\title{
GENERAL FRACTIONAL SOBOLEV SPACE WITH VARIABLE EXPONENT AND APPLICATIONS TO NONLOCAL PROBLEMS
}

\author{
E. AZROUL ${ }^{1}$, A. BENKIRANE ${ }^{2}$ AND M. SHIMI ${ }^{3}$
}

\begin{abstract}
In this paper, we extend the fractional Sobolev spaces with variable exponents $W^{s, p(x, y)}$ to include the general fractional case $W^{K, p(x, y)}$, where $p$ is a variable exponent, $s \in(0,1)$ and $K$ is a suitable kernel. We are concerned with some qualitative properties of the space $W^{K, p(x, y)}$ (completeness, reflexivity, separability and density). Moreover, we prove a continuous and compact embedding theorem of these spaces into variable exponent Lebesgue spaces. As applications, we discus the existence of a nontrivial solution for a nonlocal $p(x,$.$) -Kirchhoff$ type problem. Further, we establish the existence and uniqueness of a solution for a variational problem involving the integro-differential operator of elliptic type $\mathcal{L}_{K}^{p(x, .)}$.
\end{abstract}

\section{INTRODUCTION}

Our main goal in this paper is to extend the fractional Sobolev spaces with variable exponents to cover the nonlocal general case with singular kernel. For this, we begin this work by remembering the definition of fractional Sobolev spaces with variable exponent, see for instance [3, 4, 11, 15].

Let $\Omega$ be a smooth bounded open set in $\mathbb{R}^{N}$. We start by fixing $s \in(0,1)$ and let $p: \bar{\Omega} \times \bar{\Omega} \longrightarrow(1,+\infty)$ be a continuous bounded function. We assume that

$$
1<p^{-}=\min _{(x, y) \in \bar{\Omega} \times \bar{\Omega}} p(x, y) \leqslant p(x, y) \leqslant p^{+}=\max _{(x, y) \in \bar{\Omega} \times \bar{\Omega}} p(x, y)<+\infty
$$

and

$p$ is symmetric, that is, $p(x, y)=p(y, x)$ for all $(x, y) \in \bar{\Omega} \times \bar{\Omega}$.

Let denote by :

$$
\bar{p}(x)=p(x, x) \quad \text { for all } \quad x \in \bar{\Omega} .
$$

We define the fractional Sobolev space with variable exponent via the Gagliardo approach as follows,

$$
\begin{aligned}
W & =W^{s, p(x, y)}(\Omega) \\
& =\left\{u \in L^{\bar{p}(x)}(\Omega): \int_{\Omega \times \Omega} \frac{|u(x)-u(y)|^{p(x, y)}}{\lambda^{p(x, y)}|x-y|^{s p(x, y)+N}} d x d y<+\infty, \text { for some } \lambda>0\right\},
\end{aligned}
$$

2010 Mathematics Subject Classification. 46E35, 35R11, 35S05, 35J35.

Key words and phrases. Generalized fractional Sobolev spaces, Nonlocal and integro-differential operators, $p(x,$.$) -Kirchhoff type problems, mountain pass theorem, Minty-Browder theorem.$ 
where $L^{\bar{p}(x)}(\Omega)$ is the Lebesgue space with variable exponent, (see Section 2). The space $W^{s, p(x, y)}(\Omega)$ is a Banach space (see [11]) if it is endowed with the norm,

$$
\|u\|_{W^{s, p(x, y)}(\Omega)}=\|u\|_{W}=\|u\|_{L^{\bar{p}(x)}(\Omega)}+[u]_{s, p(x, y)},
$$

where $[.]_{s, p(x, y)}$ is a Gagliardo semi-norm with variable exponent, which is defined by

$$
[u]_{s, p(x, y)}=\inf \left\{\lambda>0: \int_{\Omega \times \Omega} \frac{|u(x)-u(y)|^{p(x, y)}}{\lambda^{p(x, y)}|x-y|^{s p(x, y)+N}} d x d y \leqslant 1\right\} .
$$

The space $\left(W,\|\cdot\|_{W}\right)$ is separable and reflexive, see ([5, Lemma 3.1]).

Let us consider the fractional $p(x,$.$) -Laplacian operator given by$

$$
\left(-\Delta_{p(x, .)}\right)^{s} u(x)=p . v \cdot \int_{\Omega} \frac{|u(x)-u(y)|^{p(x, y)-2}(u(x)-u(y))}{|x-y|^{n+s p(x, y)}} d y, \text { for all } x \in \Omega
$$

where $p . v$. is a commonly used abbreviation in the principal value sense. One typical feature of this operator is the nonlocality, in the sense that the value of $\left(-\Delta_{p(x, .)}\right)^{s} u(x)$ at any point $x \in \Omega$ depends not only on the values of $u$ on $\Omega$, but actually on the entire space $\mathbb{R}^{N}$.

Note that the operator $\left(-\Delta_{p(x, .)}\right)^{s}$ is the fractional version of well known $p(x)$ Laplacian operator $\Delta_{p(x)} u(x)=\operatorname{div}\left(|\nabla u(x)|^{p(x)-2} u(x)\right)$. On the other hand, we remark that in the constant exponent case it is know as the fractional $p$-Laplacian operator $(-\Delta)_{p}^{s}$. This nonlinear operator is consistent, up to some normalization constant depending upon $N$ and $s$, with the linear fractional Laplacian $(-\Delta)^{s}$ in the case $p=2$. The interest for this last operator and more generally pseudo-differential operators, has constantly increased over the last few years, although such operators have been a classical topic of functional analysis since long ago. Nonlocal operators such as $(-\Delta)^{s}$ and its generalisation $\mathcal{L}_{K}$ (see for instance [14, 16, 20, 21, 22]) naturally arise in continuum mechanics, phase transition phenomena, population dynamics and game theory, as they are the typical outcome of stochastical stabilization of Lévy processes, see e.g. [9, 18, 19]. We refer the reader to [7, 12] and to the references included for a self-contained overview of the basic properties of fractional Sobolev spaces and fractional Laplacian operator.

Now, we introduce the nonlocal integro-differential operator of elliptic type $\mathcal{L}_{K}^{p(x, .)}$ that generalizes the operator $\left(-\Delta_{p(x, .)}\right)^{s}$, as follows

$$
\begin{aligned}
\mathcal{L}_{K}^{p(x, .)}(u(x)) & =p \cdot v \cdot \int_{\mathbb{R}^{N}}|u(x)-u(y)|^{p(x, y)-2}(u(x)-u(y)) K(x, y) d y, \text { for all } x \in \mathbb{R}^{N} \\
& =\lim _{\varepsilon \longrightarrow 0} \int_{\mathbb{R}^{N} \backslash B_{\varepsilon}(x)}|u(x)-u(y)|^{p(x, y)-2}(u(x)-u(y)) K(x, y) d y, \text { for all } x \in \mathbb{R}^{N} .
\end{aligned}
$$


where p.v. is a commonly used abbreviation in the principal value sense, $p: \mathbb{R}^{N} \times$ $\mathbb{R}^{N} \longrightarrow(1,+\infty)$ is a continuous bounded function satisfy (1.1), (1.2) and

$$
p((x, y)-(z, z))=p(x, y), \text { for all }(x, y),(z, z) \in \mathbb{R}^{N} \times \mathbb{R}^{N} .
$$

The kernel $K: \mathbb{R}^{N} \times \mathbb{R}^{N} \longrightarrow(0,+\infty)$ is a measurable function with the following properties:

$$
K(x, y)=K(y, x) \text { for any }(x, y) \in \mathbb{R}^{N} \times \mathbb{R}^{N},
$$

there exists $k_{0}>0$ such that

$$
\begin{gathered}
K(x, y) \geqslant k_{0}|x-y|^{-(N+s p(x, y))}, \text { for any }(x, y) \in \mathbb{R}^{N} \times \mathbb{R}^{N} \text { and } x \neq y, \\
m K \in L^{1}\left(\mathbb{R}^{N} \times \mathbb{R}^{N}\right) \text {, where } m(x, y)=\min \left\{1,|x-y|^{p(x, y)}\right\} .
\end{gathered}
$$

A typical example for $K$ is given by singular kernel $K(x, y)=|x-y|^{-(N+s p(x, y))}$. In this case $\mathcal{L}_{K}^{p(x, .)}=\left(-\Delta_{p(x, .)}\right)^{s}$. An other example for $K$ is given by the kernel

$$
K_{1}(x, y)=|x-y|^{-(N+s p(x, y))} a(x-y),
$$

where $a: \mathbb{R}^{N} \longrightarrow[1,+\infty)$ is a bounded function, that is, $a \in L^{\infty}\left(\mathbb{R}^{N}\right)$. It is easy to see that $K_{1}$ satisfy the assumptions (1.4)-(1.6).

This paper is organized as follows. In Section 2, we give some definitions and fundamental properties of the spaces $L^{q(x)}$ and $W^{s, p(x, y)}$. In Section 3, we compare the space $W^{s, p(x, y)}$ with $W^{K, p(x, y)}$ and we study the completeness, reflexivity, separability, and density of these spaces. Moreover, we prove a continuous and compact embedding theorem of these spaces into variable exponent Lebesgue spaces. In section 4 , we prove some basic properties of the operator $\mathcal{L}_{K}^{p(x .)}$. As applications, in Section 5, we show the existence of a nontrivial solution for a nonlocal $p(x,$.$) -$ Kirchhoff type problem by means of mountain pass theorem. Finally, we apply the Minty-Browder theorem to establish the existence and uniqueness of a solution for a variational problem involving the the integro-differential operator $\mathcal{L}_{K}^{p(x, .)}$.

\section{SOME PRELIMINARY RESUltS}

In this section, we recall some necessary properties of variable exponent spaces. For more details we refer the reader to $[10,13,17]$, and the references therein.

Consider the set

$$
C_{+}(\bar{\Omega})=\{q \in C(\bar{\Omega}): q(x)>1, \text { for all } x \in \bar{\Omega}\} .
$$

For all $q \in C_{+}(\bar{\Omega})$, we define

$$
q^{+}=\sup _{x \in \bar{\Omega}} q(x) \text { and } q^{-}=\inf _{x \in \bar{\Omega}} q(x) .
$$

Such that

$$
1<q^{-} \leqslant q(x) \leqslant q^{+}<+\infty .
$$


For any $q \in C_{+}(\bar{\Omega})$, we define the variable exponent Lebesgue space as

$$
L^{q(x)}(\Omega)=\left\{u: \Omega \longrightarrow \mathbb{R} \text { measurable }: \int_{\Omega}|u(x)|^{q(x)} d x<+\infty\right\} .
$$

This vector space endowed with the Luxemburg norm, which is defined by

$$
\|u\|_{L^{q(x)}(\Omega)}=\inf \left\{\lambda>0: \int_{\Omega}\left|\frac{u(x)}{\lambda}\right|^{q(x)} d x \leqslant 1\right\}
$$

is a separable reflexive Banach space.

Let $\hat{q} \in C_{+}(\bar{\Omega})$ be the conjugate exponent of $q$, that is, $\frac{1}{q(x)}+\frac{1}{\hat{q}(x)}=1$. Then we have the following Hölder-type inequality

Lemma 2.1. (Hölder inequality). If $u \in L^{q(x)}(\Omega)$ and $v \in L^{\hat{q}(x)}(\Omega)$, so

$$
\left|\int_{\Omega} u v d x\right| \leqslant\left(\frac{1}{q^{-}}+\frac{1}{\hat{q}^{-}}\right)\|u\|_{L^{q(x)}(\Omega)}\|v\|_{L^{\hat{q}(x)}(\Omega)} \leqslant 2\|u\|_{L^{q(x)}(\Omega)}\|v\|_{L^{\hat{q}(x)}(\Omega)} .
$$

A very important role in manipulating the generalized Lebesgue spaces with variable exponent is played by the modular of the $L^{q(x)}(\Omega)$ space, which defined by

$$
\begin{aligned}
\rho_{q(.)}: L^{q(x)}(\Omega) & \longrightarrow \mathbb{R} \\
u & \longrightarrow \rho_{q(.)}(u)=\int_{\Omega}|u(x)|^{q(x)} d x
\end{aligned}
$$

Proposition 2.1. Let $u \in L^{q(x)}(\Omega)$, then we have,

(i) $\|u\|_{L^{q(x)}(\Omega)}<1(\operatorname{resp}=1,>1) \Leftrightarrow \rho_{q(.)}(u)<1(\operatorname{resp}=1,>1)$,

(ii) $\|u\|_{L^{q(x)}(\Omega)}<1 \Rightarrow\|u\|_{L^{q(x)}(\Omega)}^{q+} \leqslant \rho_{q(.)}(u) \leqslant\|u\|_{L^{q(x)}(\Omega)}^{q-}$,

(iii) $\|u\|_{L^{q(x)}(\Omega)}>1 \Rightarrow\|u\|_{L^{q(x)}(\Omega)}^{q-} \leqslant \rho_{q(.)}(u) \leqslant\|u\|_{L^{q(x)}(\Omega)}^{q+}$.

Proposition 2.2. If $u, u_{k} \in L^{q(x)}(\Omega)$ and $k \in \mathbb{N}$, then the following assertions are equivalent

(i) $\lim _{k \rightarrow+\infty}\left\|u_{k}-u\right\|_{L^{q(x)}(\Omega)}=0$,

(ii) $\lim _{k \rightarrow+\infty} \rho_{q(.)}\left(u_{k}-u\right)=0$,

(iii) $u_{k} \longrightarrow u$ in measure in $\Omega$ and $\lim _{k \rightarrow+\infty} \rho_{q(.)}\left(u_{k}\right)=\rho_{q(.)}(u)$.

From Theorems 1.6, 1.8 and 1.10 of [13], we obtain the following proposition:

Proposition 2.3. Suppose that (2.1) is satisfied. If $\Omega$ is a bounded open domain, $\left(L^{q(x)}(\Omega),\|u\|_{L^{q(x)}(\Omega)}\right)$ is a reflexive uniformly convex and separable Banach space.

Definition 2.1. Let $p: \bar{\Omega} \times \bar{\Omega} \longrightarrow(1,+\infty)$, be a continuous variable exponent and $s \in(0,1)$. For any $u \in W$, we define the modular

$\rho_{p(., .)}: W \longrightarrow \mathbb{R}$, by

$$
\rho_{p(. . .)}(u)=\int_{\Omega \times \Omega} \frac{|u(x)-u(y)|^{p(x, y)}}{|x-y|^{N+s p(x, y)}} d x d y+\int_{\Omega}|u(x)|^{\bar{p}(x)} d x
$$


and

$$
\|u\|_{\rho_{p(. . .)}}=\inf \left\{\lambda>0: \rho_{p(., .)}\left(\frac{u}{\lambda}\right) \leqslant 1\right\}
$$

\section{Remark 2.1.}

(i) It is easy to see that $\|.\|_{\rho_{p(., .)}}$ is a norm on $W$ which is equivalent to the norm $\|\cdot\|_{W}$.

(ii) $\rho_{p(. . .)}$ also check the results of Propositions 2.1 and 2.2.

The following inequality can be easily obtained.

Lemma 2.2. Let $p: \bar{\Omega} \times \bar{\Omega} \longrightarrow(1,+\infty)$, be a continuous variable exponent and $s \in(0,1)$. For any $u \in W_{0}$, we have

$$
\begin{aligned}
& \text { (i) } 1 \leqslant[u]_{s, p(x, y)} \Rightarrow[u]_{s, p(x, y)}^{p^{-}} \leqslant \int_{\Omega \times \Omega} \frac{|u(x)-u(y)|^{p(x, y)}}{|x-y|^{N+s p(x, y)}} d x d y \leqslant[u]_{s, p(x, y)}^{p^{+}}, \\
& \text {(ii) }[u]_{s, p(x, y)} \leqslant 1 \Rightarrow[u]_{s, p(x, y)}^{p^{+}} \leqslant \int_{\Omega \times \Omega} \frac{|u(x)-u(y)|^{p(x, y)}}{|x-y|^{N+s p(x, y)}} d x d y \leqslant[u]_{s, p(x, y)}^{p^{-}} .
\end{aligned}
$$

In [15], the authors introduce the variable exponent Sobolev fractional space as follows

$$
\begin{aligned}
E & =W^{s, q(x), p(x, y)}(\Omega) \\
& =\left\{u \in L^{q(x)}(\Omega): \int_{\Omega \times \Omega} \frac{|u(x)-u(y)|^{p(x, y)}}{\lambda^{p(x, y)}|x-y|^{s p(x, y)+N}} d x d y<+\infty, \text { for some } \lambda>0\right\},
\end{aligned}
$$

where $q: \bar{\Omega} \longrightarrow(1,+\infty)$ be a continuous function satisfying (2.1).

We would like to mention that the continuous and compact embedding theorem has been proved in [15] under the assumption $q(x)>\bar{p}(x)=p(x, x)$. The authors in [4] give a slightly different version of continuous compact embedding theorem assuming that $q(x)=\bar{p}(x)=p(x, x)$..

Theorem 2.1. ([4]). Let $\Omega$ be a Lipschitz bounded domain in $\mathbb{R}^{N}$ and let $s \in(0,1)$. Let $p: \bar{\Omega} \times \bar{\Omega} \longrightarrow(1,+\infty)$ be a continuous function satisfies (1.1) and (1.2) with $s p^{+}<N$. Let $r: \bar{\Omega} \longrightarrow(1,+\infty)$ be a continuous variable exponent such that

$$
1<r^{-}=\min _{x \in \bar{\Omega}} r(x) \leqslant r(x)<p_{s}^{*}(x)=\frac{N \bar{p}(x)}{N-s \bar{p}(x)} \text { for all } x \in \bar{\Omega} .
$$

Then, there exists a constant $C=C(N, s, p, r, \Omega)>0$ such that, for any $u \in W$,

$$
\|u\|_{L^{r(x)}(\Omega)} \leqslant C\|u\|_{W} .
$$

That is, the space $W$ is continuously embedded in $L^{r(x)}(\Omega)$. Moreover, this embedding is compact.

Remark 2.2. Let $W_{0}$ denotes the closure of $C_{0}^{\infty}(\Omega)$ in $W$, that is,

$$
W_{0}=\bar{C}_{0}^{\infty}(\Omega){ }^{\|\cdot\|_{W}} .
$$

(i) Theorem 2.1 remains true if we replace $W$ by $W_{0},(\Omega$ is not necessarily smooth). 
(ii) Since $1<p^{-} \leqslant \bar{p}(x)<p_{s}^{*}(x)$, for any $x \in \bar{\Omega}$, then Theorem 2.1 implies that $[.]_{s, p(x, y)}$ is a norm on $W_{0}$, which is equivalent to the norm $\|\cdot\|_{W}$. So $\left(W_{0},[\cdot]_{s, p(x, y)}\right)$ is a Banach space.

Let denote by the $\mathcal{L}$ the operator associated to the $\left(-\Delta_{p(x, .)}\right)^{s}$ defined as

$$
\begin{aligned}
\mathcal{L}: W_{0} & \longrightarrow W_{0}^{*} \\
u & \longrightarrow \mathcal{L}(u): W_{0} \longrightarrow \mathbb{R} \\
\varphi & \longrightarrow<\mathcal{L}(u), \varphi>
\end{aligned}
$$

such that

$$
<\mathcal{L}(u), \varphi>=\int_{\Omega \times \Omega} \frac{|u(x)-u(y)|^{p(x, y)-2}(u(x)-u(y))(\varphi(x)-\varphi(y))}{|x-y|^{N+s p(x, y)}} d x d y,
$$

where $W_{0}^{*}$ is the dual space of $W_{0}$.

Lemma 2.3. ([5]). Assume that hypothesis (1.1) and (1.2) are satisfied and $s \in$ $(0,1)$. Then, the the following assertions hold:

- $\mathcal{L}$ is a bounded and strictly monotone operator.

- $\mathcal{L}$ is a mapping of type $\left(S_{+}\right)$, that is, if $u_{k} \rightarrow u$ in $W_{0}$ and $\limsup <\mathcal{L}\left(u_{k}\right)-\mathcal{L}(u), u_{k}-u>\leqslant 0$, then $u_{k} \longrightarrow u$ in $W_{0}$.

- $\mathcal{L}$ is a homeomorphism.

\section{FunCtional FRAMEWORK}

One of the aims of this paper is to study nonlocal problems driven by $\mathcal{L}_{K}^{p(x, .)}$ and $\left(-\Delta_{p(x, .)}\right)^{s}$ with Dirichlet boundary data via variational methods. For this purpose, we need to work in a suitable fractional Sobolev space. For this, we consider a functional analytical setting that is inspired by (but not equivalent to) the fractional Sobolev spaces in order to correctly encode the Dirichlet boundary datum in the variational formulation.

This section is devoted to the definition of this space as well as to its properties. Further, we will prove a continuous compact embedding theorem of these spaces into variable exponent Lebesgue spaces. Finally, we establish a convergence property for a bounded sequence in $W_{0}^{K, p(x, y)}(\Omega)$.

Let $\Omega$ be a Lipschitz open bounded subset of $\mathbb{R}^{N}, s \in(0,1)$ be fixed such that $s p^{+}<N$. Denote by $Q$ the set

$$
Q:=\mathbb{R}^{N} \times \mathbb{R}^{N} \backslash(C \Omega \times C \Omega), \text { where } C \Omega=\mathbb{R}^{N} \backslash \Omega .
$$

Now, due to the non-locality of the operator $\mathcal{L}_{K}^{p(x, .)}$ we introduce the general fractional Sobolev space with variable exponent as follows

$$
W^{K, p(x, y)}(\Omega)=\left\{\begin{array}{l}
u: \mathbb{R}^{N} \longrightarrow \mathbb{R} \text { measurable, such that } u_{\mid \Omega} \in L^{\bar{p}(x)}(\Omega) \text { with } \\
\int_{Q} \frac{|u(x)-u(y)|^{p(x, y)}}{\lambda^{p(x, y)}} K(x, y) d x d y<+\infty, \text { for some } \lambda>0
\end{array}\right\} .
$$


The norm in $W^{K, p(x, y)}(\Omega)$ can be defined as follows:

$$
\|u\|_{W^{K, p(x, y)}(\Omega)}=\|u\|_{K, p(x, y)}=\|u\|_{L^{\bar{p}(x)}(\Omega)}+[u]_{K, p(x, y)},
$$

where, $[u]_{K, p(x, y)}=\inf \left\{\lambda>0: \int_{Q} \frac{|u(x)-u(y)|^{p(x, y)}}{\lambda^{p(x, y)}} K(x, y) d x d y \leqslant 1\right\}$, (see Lemma $3.1)$.

For any $u \in W^{K, p(x, y)}(\Omega)$, we define the functional

$$
\rho_{K, p(., .)}(u)=\int_{Q}|u(x)-u(y)|^{p(x, y)} K(x, y) d x d y+\int_{\Omega}|u(x)|^{\bar{p}(x)} d x .
$$

It is easy to see that $\rho_{K, p(. . .)}$ is a convex modular on $W^{K, p(x, y)}(\Omega)$. The norm associated with $\rho_{K, p(. .)}$ is given by

$$
\|u\|_{\rho_{K, p(. .)}}=\inf \left\{\lambda>0: \rho_{K, p(.,)}\left(\frac{u}{\lambda}\right) \leqslant 1\right\} .
$$

Using the same argument as in [10, Theorem 2.17], we prove that $\|\cdot\|_{\rho_{K, p(., .)}}$ is a norm on $W^{K, p(x, y)}(\Omega)$, which is equivalent to the norm $\|\cdot\|_{K, p(x, y)}$. We also define the closed linear subspace of $W^{K, p(x, y)}(\Omega)$ by

$$
W_{0}^{K, p(x, y)}(\Omega)=\left\{u \in W^{K, p(x, y)}(\Omega): u(x)=0 \text { a.e. } x \in \mathbb{R}^{N} \backslash \Omega\right\} .
$$

On the other hand, for any $u \in W_{0}^{K, p(x, y)}(\Omega)$, we define the functional

$$
\rho_{K, p(., .)}^{o}(u)=\int_{Q}|u(x)-u(y)|^{p(x, y)} K(x, y) d x d y .
$$

$\rho_{K, p(., .)}^{o}$ is a convex modular on $W_{0}^{K, p(x, y)}(\Omega)$. The norm associated with $\rho_{K, p(., .)}^{o}$ is given by

$$
\|u\|_{\rho_{K, p(. .)}^{o}}=[u]_{K, p(x, y)}=\inf \left\{\lambda>0: \rho_{K, p(., .)}^{o}\left(\frac{u}{\lambda}\right) \leqslant 1\right\} .
$$

\section{Remark 3.1.}

(1) $\rho_{K, p(. .)}^{o}$ also check the results of Proposition 2.2.

(2) The modular $\rho_{K, p(. . .)}^{o}$ does not satisfy the triangle inequality, that is,

$$
\rho_{K, p(., .)}^{o}(u+v) \leqslant \rho_{K, p(., .)}^{o}(u)+\rho_{K, p(., .)}^{o}(v)
$$

However, there is a substitute that is sometimes useful.

$$
\rho_{K, p(., .)}^{o}(u+v) \leqslant 2^{p^{+}-1}\left(\rho_{K, p(., .)}^{o}(u)+\rho_{K, p(., .)}^{o}(v)\right) .
$$

We will refer to this as the modular triangle inequality.

Lemma 3.1. $\|\cdot\|_{K, p(x, y)}$ is a norm on $W^{K, p(x, y)}(\Omega)$.

Proof. Since $\|\cdot\|_{L^{\bar{p}(x)}(\Omega)}$ is a norm on $L^{\bar{p}(x)}(\Omega)$. So we need to prove that:

(i) $\|u\|_{K, p(x, y)}=0$ if and only if $u=0$,

and $[.]_{K, p(x, y)}$ is a semi-norm on $W^{K, p(x, y)}(\Omega)$, that is,

(ii) for all $\alpha \in \mathbb{R},[\alpha u]_{K, p(x, y)}=|\alpha|[u]_{K, p(x, y)}$,

(iii) $[u+v]_{K, p(x, y)} \leqslant[u]_{K, p(x, y)}+[v]_{K, p(x, y)}$. 
Indeed, For $(i)$, if $u=0$, then

$$
\int_{Q}|u(x)-u(y)|^{p(x, y)} K(x, y) d x d y=0 \quad \text { and } \quad\|u\|_{L^{\bar{p}(x)}(\Omega)}=0 .
$$

So, by Lemma 3.3 we get

$$
[u]_{K, p(x, y)}=0 \quad \text { and } \quad\|u\|_{L^{\bar{p}(x)}(\Omega)}=0 \quad \Rightarrow \quad\|u\|_{K, p(x, y)}=0 .
$$

Conversely, if $\|u\|_{K, p(x, y)}=0$, then

$$
\|u\|_{L^{\bar{p}(x)}(\Omega)}=0
$$

and

$$
[u]_{K, p(x, y)}=0 .
$$

By (3.1), we have

$$
u=0 \text { a.e. in } \Omega \text {. }
$$

Using (3.2), we get

$$
\lambda^{\star}=\inf \left\{\lambda>0: \int_{Q} \frac{|u(x)-u(y)|^{p(x, y)}}{\lambda^{p(x, y)}} K(x, y) d x d y \leqslant 1\right\}=0 .
$$

Let $\lambda_{n}>0, n \in \mathbb{N}$, such that $\lambda_{n}$ decreases to $\lambda^{\star}$ and

$$
\int_{Q} \frac{|u(x)-u(y)|^{p(x, y)}}{\lambda_{n}^{p(x, y)}} K(x, y) d x d y \leqslant 1 .
$$

If $\lambda_{n}<1$, then wa have

$$
\int_{Q} \frac{|u(x)-u(y)|^{p(x, y)}}{\lambda_{n}^{p^{-}}} K(x, y) d x d y \leqslant 1
$$

Hence

$$
\int_{Q}|u(x)-u(y)|^{p(x, y)} K(x, y) d x d y \leqslant \lambda_{n}^{p^{-}} .
$$

When $n \longrightarrow+\infty$, we obtain $\lambda_{n}^{p^{-}} \longrightarrow \lambda^{\star}=0$. Thus

$$
0 \leqslant \int_{Q}|u(x)-u(y)|^{p(x, y)} K(x, y) d x d y \leqslant 0 \Rightarrow \int_{Q}|u(x)-u(y)|^{p(x, y)} K(x, y) d x d y=0 .
$$

We conclude that $u(x)=u(y)$ a.e. $(x, y) \in Q$, then $u=c \in \mathbb{R}$ a.e. in $\mathbb{R}^{N}$. Finally, by (3.3) it easily follows that $c=0$, so $u=0$ a.e. in $\mathbb{R}^{N}$.

To prove (ii), note that if $\alpha=0$, this follows from $(i)$. Fix $\alpha \neq 0$, then by a change of 
variable, we have

$$
\begin{aligned}
{[\alpha u]_{K, p(x, y)} } & =\inf \left\{\lambda>0: \int_{Q}\left|\frac{\alpha u(x)-\alpha u(y)}{\lambda}\right|^{p(x, y)} K(x, y) d x d y \leqslant 1\right\} \\
& =\inf \left\{\lambda>0: \int_{Q} \frac{|u(x)-u(y)|^{p(x, y)}}{\left(\frac{\lambda}{|\alpha|}\right)^{p(x, y)}} K(x, y) d x d y \leqslant 1\right\} \\
& =|\alpha| \inf \left\{\frac{\lambda}{|\alpha|}>0: \int_{Q} \frac{|u(x)-u(y)|^{p(x, y)}}{\left(\frac{\lambda}{|\alpha|}\right)^{p(x, y)}} K(x, y) d x d y \leqslant 1\right\} \\
& =|\alpha| \inf \left\{\mu>0: \int_{Q} \frac{|u(x)-u(y)|^{p(x, y)}}{\mu^{p(x, y)}} K(x, y) d x d y \leqslant 1\right\} \\
& =|\alpha|[u]_{K, p(x, y)} .
\end{aligned}
$$

Finally, to prove $(i i i)$, fix $\lambda_{u}>[u]_{K, p(x, y)}$ and $\lambda_{v}>[v]_{K, p(x, y)}$. Then

$$
\rho_{K, p(., .)}^{o}\left(\frac{u}{\lambda_{u}}\right) \leqslant 1 \quad \text { and } \quad \rho_{K, p(., .)}^{o}\left(\frac{v}{\lambda_{v}}\right) \leqslant 1
$$

Now, Let $\lambda=\lambda_{u}+\lambda_{v}$, then by the convexity of $\rho_{K, p(., .)}^{o}$, we have

$$
\begin{aligned}
\rho_{K, p(., .)}^{o}\left(\frac{u+v}{\lambda}\right) & =\rho_{K, p(., .)}^{o}\left(\frac{\lambda_{u} u}{\lambda \lambda_{u}}+\frac{\lambda_{v} v}{\lambda \lambda_{v}}\right) \\
& \leqslant \frac{\lambda_{u}}{\lambda} \rho_{K, p(., .)}^{o}\left(\frac{u}{\lambda_{u}}\right)+\frac{\lambda_{v}}{\lambda} \rho_{K, p(., .)}^{o}\left(\frac{v}{\lambda_{v}}\right)
\end{aligned}
$$

Since, $\frac{\lambda_{u}}{\lambda}+\frac{\lambda_{v}}{\lambda}=1$, then

$$
\rho_{K, p(.,)}^{o}\left(\frac{u+v}{\lambda}\right) \leqslant 1
$$

Hence,

$$
[u+v]_{K, p(x, y)} \leqslant \lambda=\lambda_{u}+\lambda_{v},
$$

we take the infimum over all such $\lambda_{u}$ and $\lambda_{v}$, we get the desired inequality.

Remark 3.2. We remark that in the model case in which $K(x, y)=|x-y|^{-(N+s p(x, y))}$ the norms $\|\cdot\|_{K, p(x, y)}$ and $\|\cdot\|_{s, p(x, y)}$ are not the same, because $\Omega \times \Omega$ is strictly contained in $Q$ : This makes the fractional Sobolev space with variable exponent $W^{s, p(x, y)}(\Omega)$ not sufficient for studying the nonlocal problems.

Lemma 3.2. Let $K: \mathbb{R}^{N} \times \mathbb{R}^{N} \longrightarrow(0,+\infty)$ be a measurable function satisfy (1.4) and (1.6), let $p: \mathbb{R}^{N} \times \mathbb{R}^{N} \longrightarrow(1,+\infty)$ be a continuous bounded function satisfy (1.1) and (1.2). Then

$$
C_{0}^{\infty}(\Omega) \subset W_{0}^{K, p(x, y)}(\Omega) .
$$


Proof. Using the same argument as in [22], this lemma can be proved. For completeness, we give its proof. For $u \in C_{0}^{\infty}(\Omega)$, we only need to check that $[u]_{K, p(x, y)}<+\infty$. From Lemma 3.3, we need to prove that

$$
\int_{\mathbb{R}^{2 N}}|u(x)-u(y)|^{p(x, y)} K(x, y) d x d y<+\infty .
$$

Indeed, since $u=0$ in $\mathbb{R}^{N} \backslash \Omega$, we have that

$$
\begin{aligned}
\int_{\mathbb{R}^{2 N}}|u(x)-u(y)|^{p(x, y)} K(x, y) d x d y= & \int_{\Omega \times \Omega}|u(x)-u(y)|^{p(x, y)} K(x, y) d x d y \\
& +2 \int_{\Omega \times\left(\mathbb{R}^{N} \backslash \Omega\right)}|u(x)-u(y)|^{p(x, y)} K(x, y) d x d y \\
\leqslant & 2 \int_{\Omega \times \mathbb{R}^{N}}|u(x)-u(y)|^{p(x, y)} K(x, y) d x d y .
\end{aligned}
$$

Notice that

$|u(x)-u(y)| \leqslant\|\nabla u\|_{L^{\infty}\left(\mathbb{R}^{N}\right)}|x-y|$ and $|u(x)-u(y)| \leqslant 2\|u\|_{L^{\infty}\left(\mathbb{R}^{N}\right)}$, for all $(x, y) \in \mathbb{R}^{2 N}$.

Hence,

$$
|u(x)-u(y)|^{p(x, y)} \leqslant\|\nabla u\|_{L^{\infty}\left(\mathbb{R}^{N}\right)}^{p(x, y)}|x-y|^{p(x, y)}, \text { for all }(x, y) \in \mathbb{R}^{2 N}
$$

and

$$
|u(x)-u(y)|^{p(x, y)} \leqslant 2^{p(x, y)}\|u\|_{L^{\infty}\left(\mathbb{R}^{N}\right)}^{p(x, y)}, \text { for all }(x, y) \in \mathbb{R}^{2 N} .
$$

Thus,

$$
|u(x)-u(y)|^{p(x, y)} \leqslant\left(\|\nabla u\|_{L^{\infty}\left(\mathbb{R}^{N}\right)}^{p^{+}}+\|\nabla u\|_{L^{\infty}\left(\mathbb{R}^{N}\right)}^{p^{-}}\right)|x-y|^{p(x, y)}, \text { for all }(x, y) \in \mathbb{R}^{2 N}
$$

and

$$
|u(x)-u(y)|^{p(x, y)} \leqslant 2^{p+}\left(\|u\|_{L^{\infty}\left(\mathbb{R}^{N}\right)}^{p^{+}}+\|u\|_{L^{\infty}\left(\mathbb{R}^{N}\right)}^{p^{-}}\right), \text {for all }(x, y) \in \mathbb{R}^{2 N} .
$$

So, we have

$$
|u(x)-u(y)|^{p(x, y)} \leqslant 2^{p^{+}+1}\left(\|u\|_{C^{1}\left(\mathbb{R}^{N}\right)}^{p^{+}}+\|u\|_{C^{1}\left(\mathbb{R}^{N}\right)}^{p^{-}}\right) \min \left\{1,|x-y|^{p(x, y)}\right\} .
$$

From (3.4), we deduce that

$$
\begin{aligned}
\int_{\mathbb{R}^{2 N}}|u(x)-u(y)|^{p(x, y)} K(x, y) d x d y & =2^{p^{+}+1}\left(\|u\|_{C^{1}\left(\mathbb{R}^{N}\right)}^{p^{+}}+\|u\|_{C^{1}\left(\mathbb{R}^{N}\right)}^{p^{-}}\right) \int_{\Omega \times \mathbb{R}^{N}} m(x, y) K(x, y) d x d y \\
& \leqslant C_{1} \int_{\mathbb{R}^{N} \times \mathbb{R}^{N}} m(x, y) K(x, y) d x d y
\end{aligned}
$$

Assumption (1.6) implies that $u \in W_{0}^{K, p(x, y)}(\Omega)$.

Remark 3.3. A trivial consequence of Lemma 3.2, $W^{K, p(x, y)}(\Omega)$ and $W_{0}^{K, p(x, y)}(\Omega)$ are non-empty.

The modular $\rho_{K, p(., .)}^{o}$ check the following result, which is similar to Proposition 2.1 and Lemma 2.2. 
Lemma 3.3. Let $p: \mathbb{R}^{N} \times \mathbb{R}^{N} \longrightarrow(1,+\infty)$ be a continuous variable exponent and $K$ : $\mathbb{R}^{N} \times \mathbb{R}^{N} \longrightarrow(0,+\infty)$ is a measurable function satisfy (1.4) and (1.6). Then For any $u \in W_{0}^{K, p(x, y)}$, we have
(i) $1 \leqslant[u]_{K, p(x, y)} \Rightarrow[u]_{K, p(x, y)}^{p^{-}} \leqslant \rho_{K, p(., .)}^{o}(u) \leqslant[u]_{K, p(x, y)}^{p^{+}}$,
(ii) $[u]_{K, p(x, y)} \leqslant 1 \Rightarrow[u]_{K, p(x, y)}^{p^{+}} \leqslant \rho_{K, p(., .)}^{o}(u) \leqslant[u]_{K, p(x, y)}^{p^{-}}$.

Proof. We prove the first pair of inequalities; the proof of the second is essentially the same. Indeed, it is easy to see that, for all $\lambda \in(0,1)$, we get

$$
\lambda^{p^{+}} \rho_{K, p(., .)}^{o}(u) \leqslant \rho_{K, p(., .)}^{o}(\lambda u) \leqslant \lambda^{p^{-}} \rho_{K, p(., .)}^{o}(u)
$$

Now, if $[u]_{K, p(x, y)}>1$, then $0<\frac{1}{[u]_{K, p(x, y)}}<1$, so we have

$$
\frac{\rho_{K, p(. . .)}^{o}(u)}{[u]_{K, p(x, y)}^{p^{+}}} \leqslant \rho_{K, p(. . .)}^{o}\left(\frac{u}{[u]_{K, p(x, y)}}\right) \leqslant \frac{\rho_{K, p(. .)}^{o}(u)}{[u]_{K, p(x, y)}^{p^{-}}} .
$$

Since $\rho_{K, p(., .)}^{o}\left(\frac{u}{[u]_{K, p(x, y)}}\right)=1$, so the desired result follows.

In the following lemma we compare the spaces $W^{K, p(x, y)}$ and $W^{s, p(x, y)}$. This lemma is crucial in the proof of the continuous embedding theorem.

Lemma 3.4. Let $K: \mathbb{R}^{N} \times \mathbb{R}^{N} \longrightarrow(0,+\infty)$ be a measurable function satisfying (1.4)(1.6). Let $p: \mathbb{R}^{N} \times \mathbb{R}^{N} \longrightarrow(1,+\infty)$ be a continuous bounded function satisfying (1.1) and (1.2). Then the following assertions hold:

(i) If $u \in W^{K, p(x, y)}(\Omega)$, then $u \in W^{s, p(x, y)}(\Omega)$. Moreover,

$$
\|u\|_{s, p(x, y)} \leqslant \max \left\{1, \tilde{k}_{0}\right\}\|u\|_{K, p(x, y)},
$$

where $\tilde{k}_{0}=\tilde{k}_{0}\left(k_{0}, p^{-}, p^{+}\right)$is a positive constant. That is, the space $W^{K, p(x, y)}(\Omega)$ is continuously embedded in $W^{s, p(x, y)}(\Omega)$.

(ii) If $u \in W_{0}^{K, p(x, y)}(\Omega)$, then $u \in W^{s, p(x, y)}\left(\mathbb{R}^{N}\right)$. Moreover,

$$
\|u\|_{W^{s, p(x, y)}(\Omega)} \leqslant\|u\|_{W^{s, p(x, y)\left(\mathbb{R}^{N}\right)}} \leqslant \max \left\{1, \tilde{k}_{0}\right\}\|u\|_{K, p(x, y)}
$$

Proof . (i)- Let $\lambda>0$, for $u \in W^{K, p(x, y)}(\Omega)$ and by (1.5), we have

$$
\int_{\Omega \times \Omega} \frac{|u(x)-u(y)|^{p(x, y)}}{\lambda^{p(x, y)}|x-y|^{s p(x, y)+N}} d x d y \leqslant \int_{Q} \frac{|u(x)-u(y)|^{p(x, y)}}{\lambda^{p(x, y)}} \frac{K(x, y)}{k_{0}} d x d y .
$$

We define

and

$$
\mathcal{A}_{\lambda, \Omega}^{s}=\left\{\lambda>0: \int_{\Omega \times \Omega} \frac{|u(x)-u(y)|^{p(x, y)}}{\lambda^{p(x, y)}|x-y|^{s p(x, y)+N}} d x d y \leqslant 1\right\}
$$

$$
\mathcal{A}_{\lambda, Q}^{K, k_{0}}=\left\{\lambda>0: \int_{Q} \frac{|u(x)-u(y)|^{p(x, y)}}{\lambda^{p(x, y)}} \frac{K(x, y)}{k_{0}} d x d y \leqslant 1\right\} .
$$

By (3.5), it is easy to see that $\mathcal{A}_{\lambda, Q}^{K, k_{0}} \subset \mathcal{A}_{\lambda, \Omega}^{s}$. Hence $\inf _{\lambda>0} \mathcal{A}_{\lambda, \Omega}^{s} \leqslant \inf _{\lambda>0} \mathcal{A}_{\lambda, Q}^{K, k_{0}}$. Then, we have

$$
[u]_{s, p(x, y)}=\inf _{\lambda>0} \mathcal{A}_{\lambda, \Omega}^{s} \leqslant \inf _{\lambda>0} \mathcal{A}_{\lambda, Q}^{K, k_{0}} .
$$


Now, let

And we set

$$
\mathcal{A}_{\lambda, Q}^{K}=\left\{\lambda>0: \int_{Q} \frac{|u(x)-u(y)|^{p(x, y)}}{\lambda^{p(x, y)}} K(x, y) d x d y \leqslant 1\right\} .
$$

So, we obtain

$$
\tilde{k}_{0}=\max \left\{k_{0}^{-\frac{1}{p^{-}}}, k_{0}^{-\frac{1}{p^{+}}}\right\} \quad \text { and } \quad \bar{\lambda}=\lambda \tilde{k}_{0}
$$

$$
\begin{aligned}
\tilde{k}_{0} \inf _{\lambda>0} \mathcal{A}_{\lambda, Q}^{K} & =\inf _{\lambda>0}\left\{\lambda \tilde{k}_{0}: \int_{Q} \frac{|u(x)-u(y)|^{p(x, y)}}{\lambda^{p(x, y)}} K(x, y) d x d y \leqslant 1\right\} \\
& =\inf _{\bar{\lambda}>0}\left\{\bar{\lambda}: \int_{Q} \frac{|u(x)-u(y)|^{p(x, y)}}{\bar{\lambda}^{p(x, y)}} \tilde{k}_{0}^{p(x, y)} K(x, y) d x d y \leqslant 1\right\}
\end{aligned}
$$

Since $\tilde{k}_{0} \geqslant k_{0}^{-\frac{1}{p(x, y)}}$, then $\tilde{k}_{0}^{p(x, y)} \geqslant \frac{1}{k_{0}}$. So, we get

$$
\int_{Q} \frac{|u(x)-u(y)|^{p(x, y)}}{\lambda^{p(x, y)}} \frac{K(x, y)}{k_{0}} d x d y \leqslant \int_{Q} \frac{|u(x)-u(y)|^{p(x, y)}}{\lambda^{p(x, y)}} \tilde{k}_{0}^{p(x, y)} K(x, y) d x d y
$$

Let

$$
\mathcal{B}_{\lambda, Q}^{K, \tilde{k}_{0}}=\left\{\lambda>0: \int_{Q} \frac{|u(x)-u(y)|^{p(x, y)}}{\lambda^{p(x, y)}} \tilde{k}_{0}^{p(x, y)} K(x, y) d x d y \leqslant 1\right\} .
$$

We remark that $\mathcal{B}_{\lambda, Q}^{K, \tilde{k}_{0}} \subset \mathcal{A}_{\lambda, Q}^{K, k_{0}}$. This implies that $\inf _{\lambda>0} \mathcal{A}_{\lambda, Q}^{K, k_{0}} \leqslant \inf _{\lambda>0} \mathcal{B}_{\lambda, Q}^{K, \tilde{k}_{0}}$.

Using (3), we get

$$
\inf _{\lambda>0} \mathcal{A}_{\lambda, Q}^{K, k_{0}} \leqslant \inf _{\lambda>0} \mathcal{B}_{\lambda, Q}^{K, \tilde{k}_{0}}=\tilde{k}_{0} \inf _{\lambda>0} \mathcal{A}_{\lambda, Q}^{K}
$$

Hence,by (3.6), we have

$$
[u]_{s, p(x, y)} \leqslant \tilde{k}_{0}[u]_{K, p(x, y)}<+\infty .
$$

In fact, by using the definition of norms $\|u\|_{s, p(x, y)}$ and $\|u\|_{K, p(x, y)}$, we infer

$$
\|u\|_{s, p(x, y)} \leqslant \max \left\{1, \tilde{k}_{0}\right\}\|u\|_{K, p(x, y)} .
$$

The first assertion is proved.

(ii)- For $u \in W_{0}^{K, p(x, y)}(\Omega)$, we have $u=0$ a.e. in $\mathbb{R}^{N} \backslash \Omega$. Then

$$
\|u\|_{L^{\bar{p}(x)}(\Omega)}=\|u\|_{L^{\bar{p}(x)}\left(\mathbb{R}^{N}\right)}<+\infty .
$$

By the same argument in the assertion $(i)$, we get $u \in W^{s, p(x, y)}\left(\mathbb{R}^{N}\right)$ and

$$
[u]_{W^{s, p(x, y)}(\Omega)} \leqslant[u]_{W^{s, p(x, y)}\left(\mathbb{R}^{N}\right)} \leqslant \tilde{k}_{0}[u]_{K, p(x, y)} .
$$

So the estimate on the norm is easily follows.

Now, we are ready to prove the main theorem of this section.

Theorem 3.1. Let $\Omega$ be a Lipschitz bounded domain in $\mathbb{R}^{N}$ and $s \in(0,1)$. Let $p$ : $\mathbb{R}^{N} \times \mathbb{R}^{N} \longrightarrow(1,+\infty)$ be a continuous variable exponent satisfies (1.1) and (1.2) with $s p^{+}<N$. Let $r: \bar{\Omega} \longrightarrow(1,+\infty)$ be a continuous bounded variable exponent such that

$$
1<r^{-} \leqslant r(x)<p_{s}^{*}(x), \text { for all } x \in \bar{\Omega} \text {. }
$$


Suppose that $K: \mathbb{R}^{N} \times \mathbb{R}^{N} \longrightarrow(0,+\infty)$ is a measurable function satisfying (1.4)-(1.6). Then

(i) There exists a positive constant $C=C(N, p, r, s, \Omega)>0$, such that for any $u \in$ $W^{K, p(x, y)}(\Omega)$, we have

$$
\|u\|_{L^{r(x)}(\Omega)} \leqslant C\|u\|_{W^{s, p(x, y)}(\Omega)} \leqslant C \max \left\{1, \tilde{k}_{0}\right\}\|u\|_{K, p(x, y)},
$$

That is, the space $W^{K, p(x, y)}(\Omega)$ is continuously embedded in $L^{r(x)}(\Omega)$. Moreover, this embedding is compact.

(ii) There exists a positive constant $C_{0}=C_{0}\left(N, p, s, \tilde{k}_{0}, \Omega\right)>0$, such that

$$
[u]_{K, p(x, y)} \leqslant\|u\|_{K, p(x, y)} \leqslant C_{0}[u]_{K, p(x, y)} .
$$

Proof. (i)- Let $u \in W^{K, p(x, y)}(\Omega)$, by Lemme 3.4, we have $u \in W^{s, p(x, y)}(\Omega)$ and

$$
\|u\|_{W^{s, p(x, y)}}=\|u\|_{s, p(x, y)} \leqslant \max \left\{1, \tilde{k}_{0}\right\}\|u\|_{K, p(x, y)} .
$$

Combining (3.8) with Theorem 2.1, we obtain

$$
\|u\|_{L^{r(x)}(\Omega)} \leqslant C\|u\|_{W^{s, p(x, y)}(\Omega)} \leqslant C \max \left\{1, \tilde{k}_{0}\right\}\|u\|_{K, p(x, y)} .
$$

Hence, we deduce that

$$
W^{K, p(x, y)}(\Omega) \hookrightarrow W^{s, p(x, y)}(\Omega) \hookrightarrow \hookrightarrow L^{r(x)}(\Omega) .
$$

Since the latter embedding is compact, then the embedding $W^{K, p(x, y)}(\Omega) \hookrightarrow L^{r(x)}(\Omega)$ is also compact.

(ii)- This assertion is easily follows by combining the definition of $\|\cdot\|_{K, p(x, y)}$ with assertion $(i)$ and assumptions (1.4)-(1.6).

\section{Remark 3.4.}

(1) The assertion $(i)$ implies also that $W_{0}^{K, p(x, y)}(\Omega)$ is continuously embedded in $L^{r(x)}(\Omega)$, where $1<r^{-} \leqslant r(x)<p_{s}^{*}(x)$ for any $x \in \bar{\Omega}$. Moreover, this embedding is compact.

(2) As a consequence of assertion (ii), [. $]_{K, p(x, y)}$ is an equivalent norm of $\|u\|_{K, p(x, y)}$ on $W_{0}^{K, p(x, y)}(\Omega)$.

Lemma 3.5. $\left(W_{0}^{K, p(x, y)}(\Omega),[.]_{K, p(x, y)}\right)$ is a separable, reflexive, and uniformly convex Banach space.

Proof. We first prove that $W_{0}^{K, p(x, y)}(\Omega)$ is complete with respect to the norm [.] $]_{K, p(x, y)}$. Let $\left\{u_{n}\right\}$ be a Cauchy sequence in $W_{0}^{K, p(x, y)}(\Omega)$. Since $\bar{p}(x)<p_{s}^{*}(x)$, so, combining $(i)$ and (ii) of Theorem 3.1, for any $\varepsilon>0$, there exists $n_{\varepsilon}^{\star}$ such that if $n, m \geqslant n_{\varepsilon}^{\star}$, we get

$$
\frac{1}{\bar{C}}\left\|u_{n}-u_{m}\right\|_{L^{\bar{p}(x)}(\Omega)} \leqslant\left[u_{n}-u_{m}\right]_{K, p(x, y)} \leqslant \varepsilon,
$$

where $\bar{C}=C_{0} C \max \left\{1, \tilde{k}_{0}\right\}$. By the completeness of $L^{\bar{p}(x)}(\Omega)$, there exists $u \in L^{\bar{p}(x)}(\Omega)$ such that $u_{n} \longrightarrow u$ strongly in $L^{\bar{p}(x)}(\Omega)$ as $n \longrightarrow+\infty$. Since $u_{n}=0$ a.e. in $\mathbb{R}^{N} \backslash \Omega$, so we define $u=0$ a.e. in $\mathbb{R}^{N} \backslash \Omega$. Then $u_{n} \longrightarrow u$ strongly in $L^{\bar{p}(x)}\left(\mathbb{R}^{N}\right)$ as $n \longrightarrow+\infty$. So there exists a subsequence $\left\{u_{n_{j}}\right\}$ of $\left\{u_{n}\right\}$ in $W_{0}^{K, p(x, y)}(\Omega)$, such that $u_{n_{j}} \longrightarrow u$ a.e. in $\mathbb{R}^{N}$. Now, we need to prove that $[u]_{K, p(x, y)}<+\infty$. By Lemma 3.3, is enough to show that

$$
\rho_{K, p(. . .)}^{o}(u)<+\infty .
$$


Indeed, by the Fatou Lemma, with $\varepsilon=1$, we have

$$
\begin{aligned}
\rho_{K, p(., .)}^{o}(u) & =\int_{Q}|u(x)-u(y)|^{p(x, y)} K(x, y) d x d y \\
& \leqslant \liminf _{j \longrightarrow+\infty} \int_{Q}\left|u_{n_{j}}(x)-u_{n_{j}}(y)\right|^{p(x, y)} K(x, y) d x d y \\
& \leqslant \liminf _{j \longrightarrow+\infty} \int_{Q}\left|u_{n_{j}}(x)-u_{n_{j}}(y)-\left(u_{n_{1}^{\star}}(x)-u_{n_{1}^{\star}}(y)\right)+\left(u_{n_{1}^{\star}}(x)-u_{n_{1}^{\star}}(y)\right)\right|^{p(x, y)} K(x, y) d x d y \\
& \leqslant \liminf _{j \longrightarrow+\infty} \int_{Q} 2^{p^{+}-1}\left(\left|u_{n_{j}}(x)-u_{n_{j}}(y)-\left(u_{n_{1}^{\star}}(x)-u_{n_{1}^{\star}}(y)\right)\right|^{p(x, y)}\right. \\
& \leqslant 2^{p^{+}-1} \liminf _{j \longrightarrow+\infty}\left\{\int_{Q}\left|\left(u_{n_{1}^{\star}}(x)-u_{n_{1}^{\star}}(y)\right)\right|^{p(x, y)}\right) K(x, y) d x d y \\
& \leqslant 2^{p^{+}-1}\left\{\liminf _{j \longrightarrow+\infty} \rho_{K, p(. .)}^{o}\left(u_{n_{j}}-u_{Q} \mid u_{n_{1}^{\star}}(x)+\rho_{K, p(. .)}^{o}\left(u_{n_{1}^{\star}}\right)\right\} .\right.
\end{aligned}
$$

Using Lemma 3.3 we obtain

$\rho_{K, p(., .)}^{o}(u) \leqslant 2^{p^{+}-1}\left\{\liminf _{j \longrightarrow+\infty}\left(\left[u_{n_{j}}-u_{n_{1}^{\star}}\right]_{K, p(x, y)}^{p^{+}}+\left[u_{n_{j}}-u_{n_{1}^{\star}}\right]_{K, p(x, y)}^{p^{-}}\right)+\left(\left[u_{n_{1}^{\star}}\right]_{K, p(x, y)}^{p^{+}}+\left[u_{n_{1}^{\star}}\right]_{K, p(x, y)}^{p^{-}}\right)\right\}$.

By the inequality (3.9) with $\varepsilon=1$, we get

$$
\rho_{K, p(. . .)}^{o}(u) \leqslant 2^{p^{+}-1}\left(2+\left[u_{n_{1}^{\star}}\right]_{K, p(x, y)}^{p^{+}}+\left[u_{n_{1}^{\star}}\right]_{K, p(x, y)}^{p^{-}}\right)<+\infty .
$$

Thus $u \in W_{0}^{K, p(x, y)}(\Omega)$.

On the other hand, let $n \geqslant n_{\varepsilon}^{\star}$, combining (3.9) with Lemma 3.3 and the Fatou Lemma, we have

$$
\rho_{K, p(. . .)}^{o}\left(u_{n}-u\right) \leqslant \liminf _{j \longrightarrow+\infty} \rho_{K, p(. . .)}^{o}\left(u_{n}-u_{n_{j}}\right) \leqslant \frac{\varepsilon^{p^{+}}+\varepsilon^{p-}}{2}=\varepsilon^{\prime} .
$$

Hence,

$$
\lim _{n \longrightarrow+\infty} \rho_{K, p(. . .)}^{o}\left(u_{n}-u\right)=0 .
$$

Using Remark 3.1-(1), we conclude that

$$
\lim _{n \longrightarrow+\infty}\left[u_{n}-u\right]_{K, p(x, y)}=0 .
$$

That is, $u_{n} \longrightarrow u$ strongly in $W_{0}^{K, p(x, y)}(\Omega)$, as $n \longrightarrow+\infty$.

Let us now prove that the space $W_{0}^{K, p(x, y)}(\Omega)$ is a separable and uniformly convex reflexive space. For this, we define the operator

$$
\begin{aligned}
\mathcal{P}: W_{0}^{K, p(x, y)}(\Omega) & \longrightarrow L^{p(x, y)}(Q, d x d y) \\
u & \longrightarrow(u(x)-u(y)) K(x, y)^{\frac{1}{p(x, y)}}
\end{aligned}
$$


Clearly $\mathcal{P}$ is an isometry from $W_{0}^{K, p(x, y)}(\Omega)$ into $L^{p(x, y)}(Q)$. Since $W_{0}^{K, p(x, y)}(\Omega)$ is a Banach space, then $\mathcal{P}\left(W_{0}^{K, p(x, y)}(\Omega)\right)$ is a closed subset of $L^{p(x, y)}(Q)$ (which is a separable and reflexive uniformly convex space, see Proposition 2.3). It follows that $\mathcal{P}\left(W_{0}^{K, p(x, y)}(\Omega)\right)$ is separable and reflexive uniformly convex space. Consequently, $W_{0}^{K, p(x, y)}(\Omega)$ is also a separable and reflexive uniformly convex space.

This concludes the proof.

\section{Corollary 3.1.}

(i) $\left(W^{K, p(x, y)}(\Omega),\|\cdot\|_{K, p(x, y)}\right)$ is a separable and reflexive uniformly convex space.

(ii) If $\Omega \subset \mathbb{R}^{N}$ is a domain of class $C^{0,1}$, then $\left(W^{K, p(x, y)}(\Omega),\|\cdot\|_{K, p(x, y)}\right)$ is a Banach space.

Proof. (i)- We consider the operator

$$
\begin{aligned}
\widetilde{\mathcal{P}}: W^{K, p(x, y)}(\Omega) & \longrightarrow L^{\bar{p}}(\Omega) \times L^{p(x, y)}(Q, d x d y)=E \\
u & \longrightarrow\left(u(x),(u(x)-u(y)) K(x, y)^{\frac{1}{p(x, y)}}\right),
\end{aligned}
$$

which is an isometry from $W^{K, p(x, y)}(\Omega)$ to $E$. The rest of proof is similar to Lemma 3.5. (ii)- Since $\Omega$ of class $C^{0,1}$. Then, by the same way in [6, Theorem 2.1], we can prove that $\Omega$ is a $W^{K, p(x, y)}$-extension domain. So, for any $u \in W^{K, p(x, y)}(\Omega)$ we define the extension function $\widetilde{u}$ by

$$
\widetilde{u}(x)=\left\{\begin{array}{ccc}
u(x) & \text { if } & x \in \Omega, \\
0 & \text { if } & x \in \mathbb{R}^{N} \backslash \Omega .
\end{array}\right.
$$

The rest of proof is similar to Lemma 3.5.

In the following lemma we prove a convergence property for a bounded sequence in $W_{0}^{K, p(x, y)}(\Omega)$.

Lemma 3.6. Under the same assumptions of Theorem 3.1. And let $\left\{u_{j}\right\}$ be a bounded sequence in $W_{0}^{K, p(x, y)}(\Omega)$. Then there exists $u \in L^{r(x)}\left(\mathbb{R}^{N}\right)$, with $u=0$ a.e in $\mathbb{R}^{N} \backslash \Omega$, such that up to a subsequence

$$
u_{n} \longrightarrow u \text { strongly in } L^{r(x)}(\Omega) \text {, as } n \longrightarrow+\infty \text {. }
$$

Proof. Since $u_{j} \in W_{0}^{K, p(x, y)}(\Omega)$, then Lemma 3.4-(ii) implies that $u_{j} \in W^{s, p(x, y)}\left(\mathbb{R}^{N}\right)$, hence $u_{j} \in W^{s, p(x, y)}(\Omega)$. Moreover, by Lemma 3.4-(ii), Theorem 3.1-(ii) and the definition of $W_{0}^{K, p(x, y)}(\Omega)$, we have

$$
\left\|u_{j}\right\|_{W^{s, p(x, y)}(\Omega)} \leqslant\left\|u_{j}\right\|_{W^{s, p(x, y)}\left(\mathbb{R}^{N}\right)} \leqslant C_{0}\left[u_{j}\right]_{K, p(x, y)} .
$$

Using this fact and since $\left\{u_{j}\right\}$ is bounded in $W_{0}^{K, p(x, y)}(\Omega)$, we get that $\left\{u_{j}\right\}$ is bounded in $W^{s, p(x, y)}(\Omega)$. By Theorem 2.1, there exists $u \in L^{r(x)}(\Omega)$, such that up to a subsequence $u_{n} \longrightarrow u$ strongly in $L^{r(x)}(\Omega)$. Since $u_{j}=0$ a.e. in $\mathbb{R}^{N} \backslash \Omega$, we can define $u=0$ a.e. in $\mathbb{R}^{N} \backslash$ $\Omega$.

As in the classic case with $s$ being an integer, any function in the fractional Sobolev space $W^{K, p(x, y)}(\Omega)$ can be approximated by a sequence of smooth functions with compact support. 
Lemma 3.7. Let (1.1), (1.2) and (1.3) be satisfied. Then the space $C_{0}^{\infty}\left(\mathbb{R}^{N}\right)$ of smooth functions with compact support is dense in $W^{K, p(x, y)}(\Omega)$.

Proof. The proof is similar to the model case $K(x, y)=|x-y|^{-(N+s p(x, y))}$, in [5, Lemma 2.3].

Remark 3.5. It is worth mentioning that our functional setting above is inspired by the pioneering works of $M$. Xiang et al. in [24] when $1<p(x, y)=p=$ constant $<+\infty$, Servadi and Valdinoci in [21,22] in which the corresponding functional framework was discussed as $p=2$.

\section{Properties of the nonlocal fractional operator $\mathcal{L}_{K}^{p(x, .)}$}

In this section we give some basic properties of the nonlocal integro-differential operator of elliptic type $\mathcal{L}_{K}^{p(x, .)}$.

Let (1.1) and (1.2) be satisfied and $K: \mathbb{R}^{N} \times \mathbb{R}^{N} \longrightarrow(0,+\infty)$ is a measurable function satisfy (1.4)-(1.6). Then

$$
\begin{aligned}
\mathcal{L}_{K}^{p(x, .)}: W_{0}^{K, p(x, y)}(\Omega) & \longrightarrow\left(W_{0}^{K, p(x, y)}(\Omega)\right)^{*} \\
u & \longrightarrow \mathcal{L}_{K}^{p(x, .)}(u): W_{0}^{K, p(x, y)}(\Omega) \\
\longrightarrow & \longrightarrow \mathbb{R} \\
\varphi & \longrightarrow<\mathcal{L}_{K}^{p(x, .)}(u), \varphi>
\end{aligned}
$$

such that

$$
<\mathcal{L}_{K}^{p(x, .)}(u), \varphi>=\int_{\mathbb{R}^{2 N}}|u(x)-u(y)|^{p(x, y)-2}(u(x)-u(y))(\varphi(x)-\varphi(y)) K(x, y) d x d y,
$$

where $\left(W_{0}^{K, p(x, y)}(\Omega)\right)^{*}$ is the dual space of $W_{0}^{K, p(x, y)}(\Omega)$.

In the following Lemma, we show some fundamental properties of the operator $\mathcal{L}_{K}^{p(x, .)}$.

Lemma 4.1. Suppose that (1.1) and (1.2) be satisfied and let $K: \mathbb{R}^{N} \times \mathbb{R}^{N} \longrightarrow(0,+\infty)$ be a measurable function satisfying (1.4)-(1.6). Then, The following assertions hold:

(i) $\mathcal{L}_{K}^{p(x, .)}$ is well defined and bounded,

(ii) $\mathcal{L}_{K}^{p(x, .)}$ is a strictly monotone operator,

(iii) $\mathcal{L}_{K}^{p(x, .)}$ is a mapping of type $\left(S_{+}\right)$, that is, if $u_{k} \rightarrow u$ in $W_{0}^{K, p(x, y)}$ and $\limsup _{k \longrightarrow+\infty}<$ $\mathcal{L}_{K}^{p(x, .)}\left(u_{k}\right)-\mathcal{L}_{K}^{p(x, .)}(u), u_{k}-u>\leqslant 0$, then $u_{k} \longrightarrow u$ in $W_{0}^{K, p(x, y)}$,

(iv) $\mathcal{L}_{K}^{p(x, .)}: W_{0}^{K, p(x, y)}(\Omega) \longrightarrow\left(W_{0}^{K, p(x, y)}(\Omega)\right)^{*}$ is a homeomorphism,

(v) $\mathcal{L}_{K}^{p(x, .)}$ is coercive.

Proof.(i)- Let $u, \varphi \in W_{0}^{K, p(x, y)}(\Omega)$. Then,

$$
\begin{aligned}
\left|<\mathcal{L}_{K}^{p(x, .)}(u), \varphi>\right| & \leqslant\left|\int_{\mathbb{R}^{2 N}}\right| u(x)-\left.u(y)\right|^{p(x, y)-2}(u(x)-u(y))(\varphi(x)-\varphi(y)) K(x, y) d x d y \mid \\
& \leqslant \int_{\mathbb{R}^{2 N}}\left(|u(x)-u(y)|^{p(x, y)-1} K(x, y)^{\frac{1}{p(x, y)}}\right)\left(|\varphi(x)-\varphi(y)| K(x, y)^{\frac{1}{p(x, y)}}\right) d x d y,
\end{aligned}
$$


where $\hat{p}: \mathbb{R}^{N} \times \mathbb{R}^{N} \longrightarrow(1,+\infty)$ is the conjugate exponent of $p$, that is, $\frac{1}{\hat{p}(x, y)}+\frac{1}{p(x, y)}=1$. If we set

$$
\begin{gathered}
\Psi(x, y)=|u(x)-u(y)|^{p(x, y)-1} K(x, y)^{\frac{1}{\hat{p}(x, y)}} \in L^{\hat{p}(x, y)}(Q, d x d y), \\
\Phi(x, y)=|\varphi(x)-\varphi(y)| K(x, y)^{\frac{1}{p(x, y)}} \in L^{p(x, y)}(Q, d x d y) .
\end{gathered}
$$

So, by Hölder inequality, we obtain

$$
\begin{aligned}
\left|<\mathcal{L}_{K}^{p(x, .)}(u), \varphi>\right| & \leqslant 2\|\Psi\|_{L^{\hat{p}(x, y)}(Q, d x d y)}\|\Phi\|_{L^{p(x, y)}(Q, d x d y)} \\
& \leqslant C\|\Phi\|_{L^{p(x, y)}(Q, d x d y)} .
\end{aligned}
$$

It follows that

$$
\left\|\mathcal{L}_{K}^{p(x, .)}(u)\right\|_{\left(W_{0}^{K, p(x, y)}(\Omega)\right)^{*} \leqslant C<+\infty .}
$$

For the proof of the properties $(i i),(i i i)$ and $(i v)$, we follow the same argument in Lemma $4.2-((i),(i i)$ and $(i i i))$ in [5].

$(v)$ - Let $u \in W_{0}^{K, p(x, y)}(\Omega)$. Then, we have

$$
<\mathcal{L}_{K}^{p(x, .)}(u), u>=\int_{\mathbb{R}^{2 N}}|u(x)-u(y)|^{p(x, y)} K(x, y) d x d y=\rho_{K, p(., .)}^{o}(u) .
$$

If $[u]_{K, p(x, y)}>1$. From Lemma 3.3-(i), we get

$$
<\mathcal{L}_{K}^{p(x, .)}(u), u>=\rho_{K, p(., .)}^{o}(u) \geqslant[u]_{K, p(x, y)}^{p^{-}}=\|u\|_{W_{0}^{K, p(x, y)}}^{p^{-}} .
$$

If $[u]_{K, p(x, y)}<1$. By Lemma 3.3-(ii), we get

$$
<\mathcal{L}_{K}^{p(x, .)}(u), u>=\rho_{K, p(., .)}^{o}(u) \geqslant[u]_{K, p(x, y)}^{p^{+}}=\|u\|_{W_{0}^{K, p(x, y)}}^{p^{+}} .
$$

Combining (4.1) and (4.2), we obtain

$$
\lim _{\|u\|_{W_{0}^{K, p(x, y)}} \longrightarrow+\infty} \frac{<\mathcal{L}_{K}^{p(x, .)}(u), u>}{\|u\|_{W_{0}^{K, p(x, y)}}}=+\infty .
$$

This concludes the proof.

\section{Application to NONLOCAL FRACTIONAL PROBlEMS With VARIABLE EXPONENT}

In this section, we work under the hypotheses of Theorem 3.1. we aim to study two problems driven by the nonlocal operator $\mathcal{L}^{p(x, .)}$ and its particular case $\left(-\Delta_{p(x, .)}\right)^{s}$

5.1. Application to Kirchhoff type problems. At first, we discus the existence of a nontrivial solution for a nonlocal $p(x,$.$) -Kirchhoff type problem of the following form$

$$
\left(\mathcal{P}_{M}^{K}\right)\left\{\begin{array}{rll}
M\left(\int_{Q} \frac{|u(x)-u(y)|^{p(x, y)}}{p(x, y)} K(x, y) d x d y\right) \mathcal{L}_{K}^{p(x, .)} u(x)+|u|^{\bar{p}(x)-2} u & =f(x, u) & \text { in } \quad \Omega, \\
u & =0 & \text { in } \quad \mathbb{R}^{N} \backslash \Omega,
\end{array}\right.
$$

where $\Omega \subset \mathbb{R}^{N}, N \geqslant 3$, is a Lipschitz bounded open domain, $M: \mathbb{R}^{+} \longrightarrow \mathbb{R}$ is a continuous function which satisfies the following polynomial growth condition 
$\left(M_{1}\right):(1-\mu) t^{\alpha(x)-1} \leqslant M(t) \leqslant(1+\mu) t^{\alpha(x)-1}$, for all $t>0$ and $\mu \in[0,1)$, with $\alpha: \bar{\Omega} \longrightarrow(1,+\infty)$ is a bounded function such that $1<\alpha^{-} \leqslant \alpha(x) \leqslant \alpha^{+}<\infty$. $f: \Omega \times \mathbb{R} \longrightarrow \mathbb{R}$ is a Carathéodory function satisfies the following growth condition

$$
\left(f_{0}\right): \quad|f(x, t)| \leqslant c_{1}\left(1+|t|^{\beta(x)-1}\right) \quad \text { for all }(x, t) \in \Omega \times \mathbb{R},
$$

where $\beta \in C_{+}(\bar{\Omega})$ such that $\beta(x)<p_{s}^{*}(x)$ for all $x \in \bar{\Omega}$, and $\frac{\beta^{-}}{\alpha^{+}}>p^{+}$.

$\left(f_{1}\right): \lim _{t \rightarrow 0} \frac{f(x, t)}{|t|^{p^{+}-1}}=0 \quad$ uniformly for $x \in \Omega$.

$(A R)$ : There exist $A>0$ and $\theta>\left(\frac{1+\mu}{1-\mu}\right) \frac{\alpha^{+}\left(p^{+}\right)^{\alpha^{+}}}{\left(p^{-}\right)^{\alpha^{-}-1}}$ such that

$$
0<\theta F(x, t)=\theta \int_{0}^{t} f(x, \tau) d \tau \leqslant f(x, t) t \quad \text { for all }|t|>A \text { and } \text { a.e. } x \in \Omega .
$$

Actually, Ambrosetti-Rabinowitz condition $(A R)$ is quite natural and important not only to ensure that the Euler-Lagrange functional has a mountain pass geometry, but also to guarantee that the boundedness of Palais-Smale (PS) sequences.

One typical feature of problem $\left(\mathcal{P}_{M}^{K}\right)$ is the nonlocality, in the sense that the value of $\left(-\Delta_{p(x, .)}\right)^{s} u(x)$ at any point $x \in \Omega$ depends not only on the values of $u$ on $\Omega$, but actually on the entire space $\mathbb{R}^{N}$. Moreover, the presence of the function $M$, which implies that the first equation in $\left(\mathcal{P}_{M}^{K}\right)$ is no longer a pointwise equation, it is no longer a pointwise identity, therefore it is often called nonlocal problem. Therefore, the Dirichlet datum is given in $\mathbb{R}^{N} \backslash \Omega$ (which is different from the classical case of the $p(x)$-Laplacian) and not simply on $\partial \Omega$. This causes some mathematical difficulties which make the study of such a problem particularly interesting. Motivated by the results in $[1,2,3,4]$, we will prove that problem $\left(\mathcal{P}_{M}^{K}\right)$ has at least one nontrivial weak solution, by means of mountain pass theorem of of Ambrosetti and Rabinowitz [23].

Throughout this part, for simplicity, we use $c_{i}$, to denote the general nonnegative or positive constant (the exact value may change from line to line), we set also $X_{0}=W_{0}^{K, p(x, y)}(\Omega)$.

Definition 5.1. We say that $u \in X_{0}$ is a weak solution of problem $\left(\mathcal{P}_{M}^{K}\right)$ if

$$
\begin{gathered}
M\left(\sigma_{p(x, y)}(u)\right) \int_{Q}|u(x)-u(y)|^{p(x, y)-2}(u(x)-u(y))(\varphi(x)-\varphi(y)) K(x, y) d x d y \\
+\int_{\Omega}|u|^{\bar{p}(x)-2} u \varphi d x-\int_{\Omega} f(x, u) \varphi(x) d x=0,
\end{gathered}
$$

for all $\varphi \in X_{0}$, where

$$
\sigma_{p(x, y)}(u)=\int_{Q} \frac{|u(x)-u(y)|^{p(x, y)}}{p(x, y)} K(x, y) d x d y .
$$

Let us consider the Euler-Lagrange functional $J: X_{0} \longrightarrow \mathbb{R}$ which associated to $\left(\mathcal{P}_{M}^{K}\right)$, and defined by

$$
\begin{aligned}
J(u) & =\widehat{M}\left(\int_{Q} \frac{|u(x)-u(y)|^{p(x, y)}}{p(x, y)} K(x, y) d x d y\right)+\int_{\Omega} \frac{1}{\bar{p}(x)}|u|^{\bar{p}(x)} d x-\int_{\Omega} F(x, u) d x \\
& =\widehat{M}\left(\sigma_{p(x, y)}(u)\right)+\int_{\Omega} \frac{1}{\bar{p}(x)}|u|^{\bar{p}(x)} d x-\int_{\Omega} F(x, u) d x,
\end{aligned}
$$


where $\widehat{M}(t)=\int_{0}^{t} M(\tau) d \tau$.

Standard arguments (see, for instance [4, Lemma 3.1]) and the continuity of $M$ imply that $J$ is well defined and $J \in C^{1}\left(X_{0}, \mathbb{R}\right)$. Moreover, for all $u, \varphi \in X_{0}$, its Gâteaux derivative is given by

$$
\begin{aligned}
<J^{\prime}(u), \varphi>=M\left(\sigma_{p(x, y)}(u)\right) & \int_{Q}|u(x)-u(y)|^{p(x, y)-2}(u(x)-u(y))(\varphi(x)-\varphi(y)) K(x, y) d x d y \\
& +\int_{\Omega}|u|^{\bar{p}(x)-2} u \varphi d x-\int_{\Omega} f(x, u) \varphi d x .
\end{aligned}
$$

Thus, the weak solutions of $\left(\mathcal{P}_{M}^{K}\right)$ coincide with the critical points of $J$.

Now, we are in a position to state our existence result as follows

Theorem 5.1. Let $\Omega$ be a Lipschitz bounded domain in $\mathbb{R}^{N}$ and let $s \in(0,1)$, let $p$ : $\bar{Q} \longrightarrow(1,+\infty)$ be a continuous function satisfies (1.1) and (1.2) with $s p^{+}<N$. Assume that the assumptions $\left(M_{1}\right),\left(f_{0}\right),\left(f_{1}\right)$ and $(A R)$ hold. Then, problem $\left(\mathcal{P}_{M}^{K}\right)$ has at least one nontrivial weak solution.

The proof of Theorem 5.1 based on mountain pass theorem of Ambrosetti and Rabinowitz, and it follows from the following Lemmas.

Lemma 5.1. Suppose that the assumptions $\left(M_{1}\right),\left(f_{0}\right)$, and $(A R)$ hold. Then, $J$ satisfies the $(P S)$ condition.

Proof. Let us assume that there exists a sequence $\left\{u_{n}\right\} \subset X_{0}$ sch that

$$
\left\{\begin{array}{l}
\left|J\left(u_{n}\right)\right| \leqslant c_{2}, \\
J^{\prime}\left(u_{n}\right) \underset{n \rightarrow+\infty}{\longrightarrow} 0 .
\end{array}\right.
$$

Using $\left(M_{1}\right),(A R)$, Proposition 2.1, Lemma 3.3 and Remark 3.4- $(i)$, for $n$ large enough, we get

$$
\begin{aligned}
c_{2}+ & \left\|u_{n}\right\|_{W_{0}} \\
\geqslant & J\left(u_{n}\right)-\frac{1}{\theta}<J^{\prime}\left(u_{n}\right), u_{n}> \\
= & \widehat{M}\left(\sigma_{p(x, y)}\left(u_{n}\right)\right)+\int_{\Omega} \frac{1}{\bar{p}(x)}|u|^{\bar{p}(x)} d x-\int_{\Omega} F\left(x, u_{n}\right) d x \\
& -\frac{1}{\theta} M\left(\sigma_{p(x, y)}\left(u_{n}\right)\right) \int_{Q}\left|u_{n}(x)-u_{n}(y)\right|^{p(x, y)} K(x, y) d x d y-\frac{1}{\theta} \int_{\Omega}\left|u_{n}\right|^{\bar{p}(x)} d x+\frac{1}{\theta} \int_{\Omega} f\left(x, u_{n}\right) u_{n} d x \\
\geqslant & \frac{1-\mu}{\alpha^{+}}\left(\sigma_{p(x, y)}\left(u_{n}\right)\right)^{\alpha(x)}-\frac{1+\mu}{\theta}\left(\sigma_{p(x, y)}\left(u_{n}\right)\right)^{\alpha(x)-1} \rho_{K, p(., .)}^{0}\left(u_{n}\right)+\left(\frac{1}{p^{+}}-\frac{1}{\theta}\right) \int_{\Omega}\left|u_{n}\right|^{\bar{p}(x)} d x \\
& \quad+\int_{\Omega}\left[\frac{1}{\theta} f\left(x, u_{n}\right) u_{n}-F\left(x, u_{n}\right)\right] d x
\end{aligned}
$$




$$
\begin{aligned}
& \geqslant \frac{1-\mu}{\alpha^{+}\left(p^{+}\right)^{\alpha^{+}}}\left(\rho_{K, p(., .)}^{0}\left(u_{n}\right)\right)^{\alpha(x)}-\frac{1+\mu}{\theta\left(p^{-}\right)^{\alpha^{-}-1}}\left(\rho_{K, p(., .)}^{0}\left(u_{n}\right)\right)^{\alpha(x)}+\left(\frac{1}{p^{+}}-\frac{1}{\theta}\right)\left\|u_{n}\right\|_{L^{\bar{p}(x)}(\Omega)}^{p^{-}} \\
& \geqslant\left(\frac{1-\mu}{\alpha^{+}\left(p^{+}\right)^{\alpha^{+}}}-\frac{1+\mu}{\theta\left(p^{-}\right)^{\alpha^{-}-1}}\right)\left\|u_{n}\right\|_{X_{0}}^{\alpha^{-} p^{-}}+\left(\frac{1}{p^{+}}-\frac{1}{\theta}\right) c_{3}\left\|u_{n}\right\|_{X_{0}}^{p^{-}} .
\end{aligned}
$$

Since $1<p^{-}<\alpha^{-} p^{-}$and $\theta>\left(\frac{1+\mu}{1-\mu}\right) \frac{\alpha^{+}\left(p^{+}\right)^{\alpha^{+}}}{\left(p^{-}\right)^{\alpha^{-}-1}}>p^{+}$, we obtain that $\left\{u_{n}\right\}$ is bounded in $X_{0}$. This information, combined with the fact that $X_{0}$ is reflexive, implies that there exists a subsequence, still denote by $\left\{u_{n}\right\}$, and $u \in X_{0}$ such that $\left\{u_{n}\right\}$ converges weakly to $u$ in $X_{0}$. Next, as $\beta(x)<p_{s}^{*}(x)$ for all $x \in \bar{\Omega}$, then by Remark 3.4-(1), $X_{0}$ is compactly embedded in $L^{\beta(x)}(\Omega)$, it follows that

$$
u_{n} \longrightarrow u \text { (strongly) in } L^{\beta(x)}(\Omega) \text { and } u_{n}(x) \longrightarrow u(x) \text { a.e. } x \in \Omega \text {. }
$$

Using (5.2), we have

$$
<J^{\prime}\left(u_{n}\right), u_{n}-u>\longrightarrow 0, \quad \text { as } n \rightarrow+\infty
$$

that is,

$M\left(\sigma_{p(x, y)}\left(u_{n}\right)\right)<\mathcal{L}_{K}^{p(x, .)}\left(u_{n}\right), u_{n}-u>+\int_{\Omega}\left|u_{n}\right|^{\bar{p}(x)-2} u_{n}\left(u_{n}-u\right) d x-\int_{\Omega} f\left(x, u_{n}\right)\left(u_{n}-u\right) d x \underset{n \rightarrow+\infty}{\longrightarrow} 0$.

From $\left(f_{0}\right)$ and Lemma 2.1, it follows that

$$
\left|\int_{\Omega} f\left(x, u_{n}\right)\left(u_{n}-u\right) d x\right| \leqslant c\left\|\left|u_{n}\right|^{\beta(x)-1}\right\|_{L^{\hat{\beta}(x)}(\Omega)}\left\|u_{n}-u\right\|_{L^{\beta(x)}(\Omega)}+c_{1} \int_{\Omega}\left|u_{n}-u\right| d x,
$$

where $\frac{1}{\beta(x)}+\frac{1}{\hat{\beta}(x)}=1$. So, by (5.3), we have

$$
\int_{\Omega} f\left(x, u_{n}\right)\left(u_{n}-u\right) d x \longrightarrow 0 \quad \text { as } n \rightarrow+\infty .
$$

Using again Hölder inequality, we obtain

$$
\int_{\Omega}|u|^{\bar{p}(x)-2} u\left(u_{n}-u\right) d x \leqslant 2\left\|u_{n}\right\|_{L^{\bar{p}(x)}(\Omega)}\left\|u_{n}-u\right\|_{L^{\bar{p}(x)}(\Omega)} .
$$

Since $u_{n}$ converges weakly to $u$ in $X_{0}$, then the compact embedding of $X_{0}$ into $L^{\bar{p}(x)}(\Omega)$ helps us to get

$$
\lim _{n \rightarrow \infty} \int_{\Omega}\left|u_{n}\right|^{\bar{p}(x)-2} u\left(u_{n}-u\right) d x=0 .
$$

Hence, by (5.4) and (5.5), we get

$$
M\left(\sigma_{p(x, y)}\left(u_{n}\right)\right)<\mathcal{L}_{K}^{p(x, y)}\left(u_{n}\right), u_{n}-u>\underset{n \rightarrow+\infty}{\longrightarrow} 0 .
$$

Now, since $\left\{u_{n}\right\}$ is bounded in $X_{0}$, we may assume that

$$
\sigma_{p(x, y)}\left(u_{n}\right) \underset{n \rightarrow+\infty}{\longrightarrow} t_{1} \geqslant 0 .
$$

If $t_{1}=0$, then $\left\{u_{n}\right\}$ converge strongly to $u=0$ in $X_{0}$ and the proof is finished.

If $t_{1}>0$, since the function $M$ is continuous, we have

$$
M\left(\sigma_{p(x, y)}\left(u_{n}\right)\right) \underset{n \rightarrow+\infty}{\longrightarrow} M\left(t_{1}\right) \geqslant 0 .
$$


Hence, by $\left(M_{1}\right)$, for $n$ large enough, we have that

$$
0<c_{4} \leqslant M\left(\sigma_{p(x, y)}\left(u_{n}\right)\right) \leqslant c_{5} .
$$

Combining (5.6) and (5.7), we deduce that

$$
<\mathcal{L}_{K}^{p(x, y)}\left(u_{n}\right), u_{n}-u>\underset{n \rightarrow+\infty}{\longrightarrow} 0 .
$$

On the other hand, since $\left\{u_{n}\right\}$ converge weakly to $u$ in $X_{0}$, we have that

$$
<J^{\prime}(u), u_{n}-u>\underset{n \rightarrow+\infty}{\longrightarrow} 0
$$

that is,

$$
M\left(\sigma_{p(x, y)}(u)\right)<\mathcal{L}_{K}^{p(x, .)}(u), u_{n}-u>+\int_{\Omega}|u|^{\bar{p}(x)-2} u\left(u_{n}-u\right) d x-\int_{\Omega} f(x, u)\left(u_{n}-u\right) d x \underset{n \rightarrow+\infty}{\longrightarrow} 0,
$$

which implies by using the same argument as before that

$$
<\mathcal{L}_{K}^{p(x, y)}(u), u_{n}-u>\underset{n \rightarrow+\infty}{\longrightarrow} 0 .
$$

Combining (5.8), and (5.9) we deduce that

$$
\limsup _{n \rightarrow+\infty}<\mathcal{L}_{K}^{p(x, y)}\left(u_{n}\right)-\mathcal{L}_{K}^{p(x, y)}(u), u_{n}-u>\leqslant 0 .
$$

By Lemma 4.1-(iii) $\mathcal{L}_{K}^{p(x, y)}$ is a mapping of type $\left(S_{+}\right)$, thus

$$
\left\{\begin{array}{c}
\limsup _{n \rightarrow+\infty}<\mathcal{L}_{K}^{p(x, y)}\left(u_{n}\right)-\mathcal{L}_{K}^{p(x, y)}(u), u_{n}-u>\leqslant 0, \\
u_{k} \rightarrow u \text { in } X_{0}, \\
\mathcal{L} \text { is a mapping of type }\left(S_{+}\right) .
\end{array} \quad \Rightarrow u_{n} \longrightarrow u \text { (strongly) in } X_{0} .\right.
$$

Consequently, $J$ satisfies the (PS) condition.

The following lemma shows that the functional $J$ satisfies the first geometrical condition of the mountain pass theorem;

Lemma 5.2. Suppose that the assumptions $\left(M_{1}\right),\left(f_{0}\right)$, and $\left(f_{1}\right)$ hold. Then there exist two positive real numbers $R$ and a such that $J(u) \geqslant a>0$ for all $u \in X_{0}$ with $\|u\|_{X_{0}}=R$.

Proof. Let $u \in X_{0}$ with $\|u\|_{X_{0}}<1$, then by $\left(M_{1}\right)$, we have

$$
J(u) \geqslant \frac{1-\mu}{\alpha^{+}\left(p^{+}\right)^{\alpha^{+}}}\|u\|_{X_{0}}^{\alpha^{+} p^{+}}+\int_{\Omega} \frac{1}{\bar{p}(x)}|u|^{\bar{p}(x)} d x-\int_{\Omega} F(x, u) d x .
$$

Since $\beta(x)<p_{s}^{*}(x)$ and $p^{+}<p_{s}^{*}(x)$ for all $x \in \bar{\Omega}$, then by Remark 3.4-(1), we have that $X_{0}$ is continuously embedded in $L^{\beta(x)}(\Omega), L^{\bar{p}(x)}(\Omega)$ and $L^{p^{+}}(\Omega)$, that is , there exist three positive constants $c_{6}, c_{7}$ and $c_{8}$ such that

$$
\|u\|_{L^{\beta(x)}(\Omega)} \leqslant c_{6}\|u\|_{X_{0}}, \quad\|u\|_{L^{p^{+}}(\Omega)} \leqslant c_{7}\|u\|_{X_{0}} \quad \text { and } \quad\|u\|_{L^{\bar{p}(x)}(\Omega)} \leqslant c_{8}\|u\|_{X_{0}} .
$$

Now, we assume that $\|u\|_{X_{0}}<\min \left\{1, \frac{1}{c_{6}}, \frac{1}{c_{8}}\right\}$, where $c_{6}$ and $c_{8}$ are the positive constant given in (5.11), then we get

$$
\|u\|_{L^{\bar{p}(x)}(\Omega)}<1 \quad \text { and } \quad\|u\|_{L^{\beta(x)}(\Omega)}<1 \text { for all } u \in X_{0} \text { with }\|u\|_{X_{0}}=R \in(0,1) .
$$


Combining $\left(f_{0}\right)$ and $\left(f_{1}\right)$, we get

$$
F(x, t) \leqslant \varepsilon|t|^{p^{+}}+c_{\varepsilon}|t|^{\beta(x)} \quad \text { for all }(x, t) \in \Omega \times \mathbb{R} .
$$

Therefore, by $\left(M_{1}\right),(5.10),(5.11)$ and Proposition 2.1-(i), we obtain

$$
\begin{aligned}
J(u) & \geqslant \frac{1-\mu}{\alpha^{+}\left(p^{+}\right)^{\alpha^{+}}}\|u\|_{X_{0}}^{\alpha^{+} p^{+}}+\frac{1}{p^{+}} \int_{\Omega}|u|^{\bar{p}(x)} d x-\varepsilon \int_{\Omega}|u|^{p^{+}} d x-c_{\varepsilon} \int_{\Omega}|u|^{\beta(x)} d x \\
& \geqslant \frac{1-\mu}{\alpha^{+}\left(p^{+}\right)^{\alpha^{+}}}\|u\|_{X_{0}}^{\alpha^{+} p^{+}}+\frac{1}{p^{+}}\|u\|_{L^{\bar{p}(x)}(\Omega)}^{p^{+}}-\varepsilon\|u\|_{L^{p^{+}}(\Omega)}^{p^{+}}-c_{\varepsilon}\|u\|_{L^{\beta(x)}(\Omega)}^{\beta^{-}} \\
& \geqslant \frac{1-\mu}{\alpha^{+}\left(p^{+}\right)^{\alpha^{+}}}\|u\|_{X_{0}}^{\alpha^{+} p^{+}}+\frac{c_{8}^{p_{+}}}{p^{+}}\|u\|_{X_{0}}^{p^{+}}-\varepsilon c_{7}^{p^{+}}\|u\|_{X_{0}}^{p^{+}}-c_{\varepsilon} c_{6}^{\beta^{-}}\|u\|_{X_{0}}^{\beta^{-}} \\
& \geqslant\left[\frac{1-\mu}{\alpha^{+}\left(p^{+}\right)^{\alpha^{+}}}\|u\|_{X_{0}}^{\alpha^{+} p^{+}-p^{+}}+\frac{c_{8}^{p_{+}}}{p^{+}}-\varepsilon c_{7}^{p^{+}}-c_{\varepsilon} c_{6}^{\beta^{-}}\|u\|_{X_{0}}^{\beta^{-}-p^{+}}\right]\|u\|_{X_{0}}^{p^{+}} .
\end{aligned}
$$

We introduce the function $g:[0,1] \longrightarrow \mathbb{R}$, defined by

$$
g(t)=\frac{1-\mu}{\alpha^{+}\left(p^{+}\right)^{\alpha^{+}}} t^{\alpha^{+} p^{+}-p^{+}}-c_{\varepsilon} c_{6}^{\beta^{-}} t^{\beta^{-}-p^{+}} .
$$

Since $\frac{\beta^{-}}{\alpha^{+}}>p^{+}$, it is clear that there exists $\bar{t} \in[0,1]$ such that

$$
\max _{t \in[0,1]} g(t)=g(\bar{t})>0 .
$$

Hence, for a fixed $\varepsilon \in\left(0, \frac{g(\bar{t})+\frac{c_{8}^{p_{+}}}{p^{+}}}{c_{7}^{p^{+}}}\right)$small enough, there exist two positive real numbers $R$ and $a$ such that $J(u) \geqslant a>0$ for all $u \in W_{0}$ with $\|u\|_{X_{0}}=R \in(0,1)$.

The following result shows that the functional $J$ satisfies the second geometrical condition of mountain pass theorem;

Lemma 5.3. Assume that $\left(M_{1}\right)$ and $(A R)$ hold. Then there exists $u_{0} \in X_{0}$ such that $\|u\|_{X_{0}}>R, J(u)<0$.

Proof. From the assumption $(A R)$, we have that

$$
F(x, t u)>t^{\theta} F(x, u) \quad \text { for all } t \geqslant 1 \text { and a.e. } x \in \Omega \text {. }
$$

Hence, by $\left(M_{1}\right)$, for $v \in X_{0}, v \neq 0$ and $t>1$, we have

$$
\begin{aligned}
J(t v) & =\widehat{M}\left(\sigma_{p(x, y)}(t v)\right)+\int_{\Omega} \frac{1}{\bar{p}(x)}|t v|^{\bar{p}(x)} d x-\int_{\Omega} F(x, t v) d x \\
& \leqslant \frac{1+\mu}{\alpha^{-}\left(p^{-}\right)^{\alpha^{-}}} t^{\alpha^{+} p^{+}}\left(\rho_{K, p(., .)}^{0}(v)\right)^{\alpha^{+}}+\frac{1}{p^{-}} t^{p^{+}} \int_{\Omega}|v|^{\bar{p}(x)} d x-t^{\theta} \int_{\Omega} F(x, v) d x .
\end{aligned}
$$

From $\theta>\left(\frac{1+\mu}{1-\mu}\right) \frac{\alpha^{+}\left(p^{+}\right)^{\alpha^{+}}}{\left(p^{-}\right)^{\alpha^{-}-1}}$, we find that $\theta>\alpha^{+} p^{+}>p^{+}$. Therefore,

$$
J(t v) \longrightarrow-\infty \text { as } t \rightarrow+\infty .
$$


Now, we are ready to prove Theorem 5.1.

Proof of Theorem 5.1. Combining Lemmas 5.1-5.3 and the fact that $J(0)=0$, we have that $J$ satisfies the assumptions of mountain pass theorem (see [23]). Therefore, $J$ has at least one nontrivial critical point, that is, problem $\left(\mathcal{P}_{M}^{K}\right)$ has at least one nontrivial weak solution.

Example 1. As a particular case we can take

- $M(t)=a+b t^{\alpha(x)-1}, \quad a, b>0$, with $\alpha: \bar{\Omega} \longrightarrow(1,+\infty)$ is a bounded function such that $1<\alpha^{-} \leqslant \alpha(x) \leqslant \alpha^{+}<\infty$.

- $K(x, y)=|x-y|^{-(N+s p(x, y))}$.

- $f(x, t)=|t|^{\gamma(x)-2} t$, where $\gamma \in C_{+}(\bar{\Omega})$ such that $\gamma(x)<p_{s}^{*}(x)$ for all $x \in \bar{\Omega}$ and $\gamma^{-}>$ $\alpha^{+} p^{+}$

In this case, problem $\left(\mathcal{P}_{M}^{K}\right)$ becomes

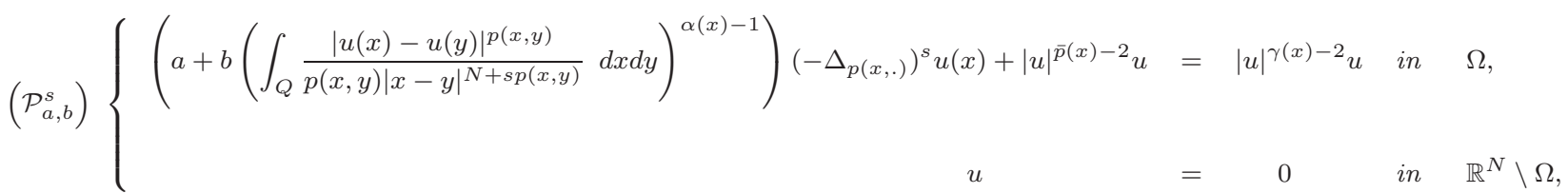

It is easy to see that the function $M$ satisfies $\left(M_{1}\right)$ and the function $f$ verify the assumptions $\left(f_{0}\right),\left(f_{1}\right)$ and the $(A R)$ conditions. Consequently, problem $\left(\mathcal{P}_{a, b}^{s}\right)$ has at least one nontrivial weak solution.

5.2. Existence and uniqueness result for a nonlocal problems. Now, we investigate the existence of a unique weak solution for a variational problem driven by general integrodifferential operators of nonlocal fractional type $\mathcal{L}_{K}^{p(x, .)}$.

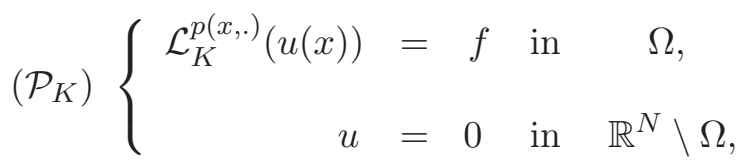

where $\Omega$ is a bounded open set of $\mathbb{R}^{N}$ and $f \in X_{0}^{*}$.

Definition 5.2. We say that $u \in X_{0}$ is a weak solution of problem $\left(\mathcal{P}_{K}\right)$, if

$$
\int_{\mathbb{R}^{2 N}}|u(x)-u(y)|^{p(x, y)-2}(u(x)-u(y))(\varphi(x)-\varphi(y)) K(x, y) d x d y=\int_{\Omega} f v d x,
$$

for any $\varphi \in X_{0}$.

Applying the Minty-Browder theorem, we get the following existence result.

Theorem 5.2. Let $\Omega$ be a bounded open set of $\mathbb{R}^{N}$ and $p: \mathbb{R}^{N} \times \mathbb{R}^{N} \longrightarrow(1,+\infty)$ be a continuous variable exponent satisfies (1.1) and (1.2) with $s p^{+}<N$. Suppose that $K$ : $\mathbb{R}^{N} \times \mathbb{R}^{N} \longrightarrow(0,+\infty)$ is a measurable function satisfying (1.4)-(1.6) and $f \in X_{0}^{*}$. Then the problem $\left(\mathcal{P}_{K}\right)$ has a unique weak solution $u \in X_{0}$.

A typical example for $K$ is given by the singular kernel $K(x, y)=|x-y|^{-(N+s p(x, y))}$. In this case, problem $\left(\mathcal{P}_{K}\right)$ becomes:

$$
\left(\mathcal{P}_{s}\right)\left\{\begin{aligned}
\left(-\Delta_{p(x, .)}\right)^{s}(u(x)) & =f \text { in } \quad \Omega \\
u & =0 \text { in } \mathbb{R}^{N} \backslash \Omega .
\end{aligned}\right.
$$


As a particular case, we derive an existence result for problem $\left(\mathcal{P}_{K}\right)$, which is given by the following corollary.

Corollary 5.1. Let $\Omega$ be a bounded open set of $\mathbb{R}^{N}$ and $p: \mathbb{R}^{N} \times \mathbb{R}^{N} \longrightarrow(1,+\infty)$ be a continuous variable exponent satisfy (1.1) and (1.2) with $s p^{+}<N$. Let $s \in(0,1)$ and $f \in X_{0}^{*}$. Then the following equation

$$
\int_{\mathbb{R}^{2 N}} \frac{|u(x)-u(y)|^{p(x, y)-2}(u(x)-u(y))(\varphi(x)-\varphi(y))}{|x-y|^{N+s p(x, y)}} d x d y=\int_{\Omega} f v d x,
$$

has a unique solution $u \in X_{0}$.

Remark 5.1. We observe that (5.13) represents the weak formulation of problem $\left(\mathcal{P}_{s}\right)$.

Proof of Theorem 5.2. By Lemma 4.1 the operator $\mathcal{L}_{K}^{p(x, .)}$ satisfies the conditions of Minty-Browder Theorem, that is,

- From Lemma 4.1-(i), $\mathcal{L}_{K}^{p(x, .)}$ is bounded, from $X_{0}$ into $X_{0}^{*}$.

- From Lemma 4.1-(ii), $\mathcal{L}_{K}^{p(x, .)}$ is a strictly monotone operator.

- From Lemma 4.1-(iv), $\mathcal{L}_{K}^{p(x, .)}$ is a homeomorphism. Hence, $\mathcal{L}_{K}^{p(x, .)}$ is continuous.

- From Lemma 4.1- $(v), \mathcal{L}_{K}^{p(x, .)}$ is coercive.

Consequently, in the light of Minty-Browder theorem [8, Theorem V.15], then there exists a unique weak solution $u \in X_{0}$ of problem $\left(\mathcal{P}_{K}\right)$.

Proof of Corollary 5.1. It is a consequence of Theorem 5.2, by choosing

$$
K(x, y)=|x-y|^{-(N+s p(x, y))},
$$

and by recalling that $X_{0} \subset W_{0}^{s, p(x, y)}(\Omega)$.

\section{REFERENCES}

[1] G.a. Afrouzi M. Mirzapour, Eigenvalue problems for $p(x)$-Kirchhoff type equations, Electronic Journal of Differential Equations, Vol. 2013 (2013), No. 253, pp. 1-10.

[2] G. A. Afrouzi, M. Mirzapour, N. T. Chung, Existence and multiplicity of solutions for a $p(x)$-Kirchhoff type equation, Rend. Semin. Mat. Univ. Padova, 136 (2016), 95-109.

[3] E. Azroul, A. Benkirane, M. Shimi and M. Srati, On a class of fractional $p(x)$-Kirchhoff type problems, Applicable Analysis, (2019), doi: 10.1080/00036811.2019.1603372

[4] E. Azroul, A. Benkirane, M. Shimi, Eigenvalue problems involving the fractional $p(x)$ Laplacian operator. Adv. Oper. Theory 4 (2019), no. 2, 539-555. doi:10.15352/aot.1809-1420.

[5] A. Bahrouni, V. Rădulescu, On a new fractional Sobolev space and applications to nonlocal variational problems with variable exponent, Discrete Contin. Dyn. Syst. 11 (2018), 379-389.

[6] A. Ballal and M. Berghout, Traces and fractional Sobolev extension Domains with variable exponent, International Journal of Mathematical Analysis, Vol. 12, no. 2 (2018), 85 - 98.

[7] G. M. Bisci, V. Rădulescu, R. Servadi, Variational methods for nonlocal fractional problems, Encyclopedia of mathematics and its applications, Cambridge University Press, Cambridge (2016).

[8] H. Brézis, Analyse fonctionnelle théorie et applications, Masson, Paris, 1983.

[9] L. Caffarelli, Nonlocal diffusions, drifts and games, in Nonlinear Partial Differential Equations, Abel Symposia 7, Springer, Berlin (2012), 37-52.

[10] D.V. Cruz-Uribe, A. Fiorenza, Variable Lebesgue spaces. Foundations and harmonic analysis. Applied and Numerical Harmonic Analysis. Birkhäuser/Springer, Heidelberg, 2013. 
[11] L. M. Del Pezzo and J. D. Rossi, Traces for fractional Sobolev spaces with variable exponents, Adv. Oper. Theory, 2 (2017), 435-446.

[12] E. Di Nezza, G. Palatucci and E. Valdinoci, Hitchhiker's guide to the fractional Sobolev spaces, Bull. Sci. Math, 136 (2012), no. 5, 521-573.

[13] X. L. Fan, D. Zhao, On the Spaces $L^{p(x)}(\Omega)$ and $W^{m, p(x)}(\Omega)$, J. Math. Anal. Appl, 263 (2001), 424-446.

[14] S. Goyal, K. Sreenadh Existence of multiple solutions of p-fractional Laplace operator with sign-changing weight function, Adv. Nonlinear Anal., 4 (2015), 37-58.

[15] U. Kaufmann, J. D. Rossi, and R. Vidal, Fractional Sobolev spaces with variable exponents and fractional $p(x)$-Laplacians, Elec. Jour. of Qual. Th, of Diff. Equa. 76 (2017), 1-10.

[16] J. Korvenpää, T. Kuusi, E. Lindgren, Equivalence of solutions to fractional p-Laplace equation, Journal de Mathématiques Pures et Appliquées, https://doi.org/10.1016/j.matpur.2017.10.004

[17] O. Kováčik, J. Rákosník, On Spaces $L^{p(x)}(\Omega)$ and $W^{m, p(x)}(\Omega)$, Czechoslovak Math. Jour. 41 (1991), No. 4, 592-618.

[18] N. Laskin, Fractional quantum mechanics and Lévy path integrals, Phys. Lett. A 268 (2000), 298-305.

[19] R. Metzler, J. Klafter, The restaurant at the random walk: recent developments in the description of anomalous transport by fractional dynamics, J. Phys. A 37 (2004), 161-208.

[20] R. Servadei, E. Valdinoci, Variational methods for non-local operators of elliptic type, discrete and continuous dynamical systems, Volume 33, Number 5, May 2013. , pp. 2105-2137

[21] R. Servadei, E. Valdinoci, Lewy-Stampacchia type estimates for variational inequalities driven by nonlocal operators, Rev. Mat. Iberoam., 29 (2013), no. 3, 1091-1126.

[22] R. Servadei and E. Valdinoci, Mountain Pass solutions for non-local elliptic operators, $J$. Math. Anal. Appl., 389 (2012), 887-898.

[23] M. Willem, Minimax Theorems, Birkhäuser, Boston, 1996.

[24] M. Q. Xiang, B. L. Zhang and M. Ferrara, Existence of solutions for Kirchhoff type problem involving the nonlocal fractional p-Laplacian, J. Math. Anal. Appl., 424 (2015), 1021-1041.

E. Azroul, A. Benkirane and M. Shimi

Sidi Mohamed Ben Abdellah University, Faculty of Sciences Dhar Al Mahraz, Laboratory of Mathematical Analysis and Applications, Fez, Morocco.

E-mail address: ${ }^{1}$ elhoussine.azroul@gmail.com

E-mail address: ${ }^{2}$ abd.benkirane@gmail.com

E-mail address: ${ }^{3}$ mohammed.shimi2@usmba.ac.ma 